\title{
V. Betriebliche Mitbestimmung 1916 bis 1934
}

\section{Der Rahmen der betrieblichen Konfliktaustragung}

Die vorhergehende Darstellung hat deutlich gemacht, daß es im Bereich der Mitbestimmung letztlich um die Etablierung eines neuen Regelsystems zur Bewältigung betrieblicher Konflikte ging. Es waren daher die betrieblichen Konflikte und die mit ihnen verbundenen Auseinandersetzungsformen, die den Mittelpunkt der Darstellung bildeten. Von diesem Punkt aus soll eine Zusammenfassung der Ergebnisse der Arbeit beginnen. Zunächst ist der Rahmen zu rekonstruieren, in dem die betrieblichen Konflikte angesiedelt waren. ${ }^{1}$

Verlauf und Ergebnisse des Ersten Weltkrieges brachten in Deutschland einen Zusammenbruch des bisher vertrauten und staatlich sanktionierten Regelsystems für das Tausch- und Vertragsverhalten der Marktparteien. Opportunismus, d.i. die Durchbrechung bislang bestehender Regeln zur Erzielung individueller Vorteile bei gleichzeitiger formaler Aufrechterhaltung und Bejahung dieser Regeln, wurde zu einem fast alltäglichen Kennzeichen des Tausch- und Vertragsverhaltens, wobei der Schwarze Markt, das Schiebertum, die Inflationsgewinnlerei, der Leistungsverfall der Arbeitsprozesse, Fabrikdiebstähle, Bummelei, um nur einige Phänomene zu nennen, dessen deutlichsten Ausdruck bildeten. Der Opportunismus bezog sich gleichermaßen auf das Marktverhalten der Unternehmen wie auf deren interne Tauschbeziehungen. Der gleichzeitige Zusammenbruch der gewohnten Markt- (Preissystem, Kartelle) wie der unternehmensinternen Tauschstrukturen (Leistungs- und Disziplinverfall) waren die zwangsläufige Folge dieses Opportunismus, der schließlich die Funktionsfähigkeit der deutschen Kriegswirtschaft ernsthaft gefährdete. Angesichts der fehlenden Kompromißbereitschaft der wirtschaftlichen Akteure waren Staat und Militär schließlich gezwungen, von sich aus neue Regelsysteme vorzuschreiben. ${ }^{2}$

Diese politische Neudefinition von Verfahrensregeln und damit auch Eigentumsrechten im Rahmen der Organisation der betrieblichen Arbeitsprozesse ${ }^{3}$ er-

1 Die Strukturierung dieser Überlegungen beruht auf den theoretischen Konzepten von North, Theorie des Institutionellen Wandels, Siegenthaler, Prosperität und Regelvertrauen, Richter, Furubotn, Institutionenökonomik und Wieland, Sozialpartnerschaft, Williamson, Mechanisms. Bis auf das Konzept von North handelt es sich hierbei nicht um historische, sondern wirtschaftstheoretische und -soziologische Konzepte. Sie werden hier ausschließlich zur Interpretation des empirischen Materials herangezogen. Eine theoretische Absicht wird nicht verfolgt, auch wenn die historische Analyse die Reichweite theoretischer Konzepte deutlich machen dürfte.

2 Eine eindrucksvolle Schilderung dieser Verfalls- und Auflösungsprozesse bei Feldman, The Great Disorder.

3 Der Ansatz der property rights oder Verfügungsrechte bezieht sich keineswegs nur auf Güter und Waren, sondern auch auf "Dienste“ und deren vertragliche Voraussetzungen und Bedingungen, die ganz maßgeblich ihren Preis bestimmen; vgl. Richter, Sichtweise und Fragestellungen, in: ZWS 110 (1994), S. $574 \mathrm{f}$. 
folgte seit 1916, vor allem aber in der sich an die Revolution anschließenden Gesetzgebungsphase. Die bei Kriegsende faktisch eingetretenen Veränderungen und die hierdurch bedingten Abmachungen der sozialen Kontrahenten, insbesondere die Vorgaben des Zentralarbeitsgemeinschaftsabkommens wurden durch ein neues, von den um Eigentumsrechte konkurrierenden Wirtschaftssubjekten freilich stark umstrittenes Regelwerk (insbesondere Tarif-, Schlichtungs- und Betriebsverfassungsrecht) kodifiziert und im Anschluß daran vom Staat sanktioniert, der aber nicht zugleich ein neues normatives Regelsystem zur Feinsteuerung des Verhaltens der Wirtschaftssubjekte schaffen konnte, das zur Kontrolle und Sanktion der neuen Regeln im alltäglichen Tauschprozeß notwendig war. Da das Regelsystem daher zunächst „nur“ auf dem Papier stand und von den Wirtschaftssubjekten je nach materieller Perspektive politisch und moralisch kritisiert wurde, dominierte zumindest während der Revolutions- und Inflationsjahre weiterhin ein moralisierter Opportunismus mit der zwangsläufig dem Opportunismus innewohnenden Tendenz zur Konflikteskalation bei wirklichen oder vermeintlichen Verlierern eines solchermaßen defekten Tausch- und Vertragsprozesses. Nach dem Ende der Inflation sanken die Möglichkeiten und auch die Vorteile des Opportunismus stark ab; es ging jetzt primär um die Nutzung der neudefinierten Eigentumsrechte zur Realisierung von Kostenvorteilen im Tausch- und Vertragsprozeß. Die Haltung zum neuen Regelwerk und ihre Überführung in einen adäquat normativ gesteuerten Tausch- und Vertragsprozeß wurde jetzt zur Funktion der im Kontext der neuen Eigentumsrechte jeweils realisierbaren Kostenvorteile. Blieben diese aus oder waren angesichts der Marktbedingungen zu gering, setzte ein erneuter Prozeß des Drängens auf politische Neudefinitionen von Eigentumsrechten ein.

Damit traten überdies andere Gesichtspunkte in den Vordergrund. Die Nutzung der neuen Eigentumsrechte und die Realisierung von Kostenvorteilen hatte neben den Marktbedingungen eine zusätzliche Determinante im Grad der erreichten technischen Differenzierung der Produktionsprozesse und ihren Auswirkungen auf die Kosten der ökonomischen Transaktionen. ${ }^{4}$ Der Inflationsschleier hatte diese für die Organisation des Arbeitsprozesses fundamentalen $\mathrm{Zu}$ sammenhänge zeitweilig vollkommen überdeckt. Mit seiner Aufhebung aber traten die "normalen“ Verhältnisse wieder ein. Das Größenwachstum der Unternehmen vor dem Ausbruch des Ersten Weltkrieges war durch eine zunehmende Funktionsinternalisierung verursacht worden, insofern Tausch- und Vertragsbeziehungen auf den Faktor- und Konsumentenmärkten, die sich aus der Sicht der beteiligten Akteure durch eine für Spotmarktorganisation zu hohe Spezifität auszeichneten, durch hierarchische Beziehungen ersetzt wurden, die sich gemessen an den Kosten der Marktoperationen als preiswerter, dauerhafter und weniger riskant darstellten. Das Größenwachstum war mithin eine Folge der relativen Preisverhältnisse zwischen den Kosten von Marktprozessen und hierarchischen Koordinationsprozessen, so wie sie sich vor allem den Unternehmensleitungen darstellten. Mit der Funktionsinternalisierung stellte sich freilich das im Unterneh-

+ Die folgenden Ausführungen folgen der Argumentation von Williamson, Die Institutionen des Kapitalismus. 
men zu bewältigende Kontrollproblem des Verhältnisses von prozessbezogenen In- und Outputs und unternehmensinterner Tauschoperationen, zu deren Kontrolle, Messung und Bewertung der Marktpreis nicht mehr zur Verfügung stand. Zwangsläufig mußten die Unternehmen daher intern wiederum kostenträchtige Kontroll- und Meßverfahren einführen, um insbesondere das Verhältnis von Arbeitsvolumen und Output, damit also die internen Transaktionskosten erfassen und ggf. senken zu können. Kontrolle und Messung von Arbeitsprozessen waren indes zwischen den beteiligten sozialen Gruppen zumindest bei der direkten Arbeitsorganisation (Akkord) umstritten, so daß sich das Problem akzeptanzfähiger Kontroll- und Meßverfahren stellte, um die Kontroll- und Meßkosten minimieren zu können. Die Ablehnung der Gewerkschaften und betrieblicher Mitbestimmung vor 1914 resultierte vor allem aus dem Bestreben, die Kontroll- und Meßkosten - abgesehen von den reinen Arbeitskosten - gering zu halten. Während nach den neudefinierten Eigentumsrechten nach 1920 eine Optimierung der Meßund Kontrollmethoden im Bereich der Produktionstechnik weiterhin in die Handlungsautonomie der Unternehmensleitungen fiel und damit hier Potentiale zur Transaktionskostensenkung eigenständig gehandhabt werden konnten, waren die Kontroll- und Meßfragen im Kontext der Arbeitsorganisation nicht nur de facto weiterhin umstritten, sondern auch de jure entfiel nunmehr die Handlungsautonomie, oder wenn man so will, das zumindest formal unbeschränkte Eigentums- und Verfügungsrecht der Unternehmensleitungen in dieser Frage. Ein Vorteil der Internalisierung von Arbeitsmärkten und ihrer Substituierung durch hierarchische Koordination von Handlungen, die ansonsten jeweils durch Marktverträge hätten geregelt werden müssen, bestand ja gerade in der Beseitigung von Vertrags- und Aushandlungskosten, die bei jeweils neuen Vertragsabschlüssen angefallen wären. In der Logik der Unternehmensentwicklung des Kaiserreiches waren die Betriebsverfassungsbestimmungen von 1916 bis 1920 daher kostentreibend bzw. behinderten die Realisierung von Kostenvorteilen, die die Substituierung von Marktprozessen begründet hatten. Da jedoch die Kontroll- und Meßfrage auch im Kaiserreich umstritten und kostentreibend war, mithin also versteckte oder offene Geschäftsführungskosten aus der Bewältigung un- bzw. wenig regulierter Konflikte (z. B. Arbeitskräftefluktuation) anfielen, bedeuteten die neuen Eigentumsrechte nicht zwangsläufig eine Tendenz zur Kostensteigerung, sondern konnten das gegenteilige Ergebnis hervorrufen, durch Erleichterung der Kontrolle und Messung der Effizienz der Arbeitsprozesse die Geschäftsführungskosten stärker zu senken, als parallel wegen der neuen Aushandlungsstruktur die Kosten stiegen. ${ }^{5}$

Kurz: Vor dem Hintergrund krisenhafter Marktveränderungen und dem Zerfall der formellen und informellen Verhaltensregeln des Kaiserreiches definierte das seit 1916 nach und nach geschaffene Arbeitsrecht ein neues Regelsystem als Grundlage einer veränderten betrieblichen Governance-Struktur, das aus der Perspektive des Kaiserreiches vordergründig kostentreibend, aus der Sicht der inter-

5 Dieser Zusammenhang ist in neueren institutionenökonomischen Untersuchungen nachhaltig betont worden, vgl. insbesondere die Arbeiten von Josef Wieland, hier Wieland, Sozialpartnerschaft, in: Hutter (Hg.), Wittener Jahrbuch für ökonomische Literatur, S. 143-160. 
nen Kontroll- und Regulierungszwänge der Unternehmen aber auch kostensenkend wirken konnte. Für die handelnden Akteure waren diese Verhältnisse nicht ohne weiteres durchschaubar, sondern mußten in einem komplizierten Verlernund Lernprozeß erst transparent gemacht werden. Der zunächst heftige Streit um die Neudefinition von Eigentums- und Verfügungsrechten behinderte nüchternes Lernen erheblich. Es mußte auf den betrieblichen Konfliktalltag nach der Inflation ankommen, ob die Vorteile einer kooperativ angelegten Konfliktregulierung begriffen und realisiert wurden.

\section{Struktur und Inhalt der betrieblichen Konflikte}

Struktur und Inhalt der betrieblichen Konflikte und damit der betrieblichen Lernprozesse ergaben sich im Rahmen der bestehenden Marktverhältnisse und Organisationsstrukturen einerseits aus den Arbeitsprozessen, zweitens aus den situativ variierenden Interessen der jeweils beteiligten betrieblichen Gruppen, drittens schließlich aus ihren Durchsetzungsstrategien und den dabei jeweils mobilisierbaren Machtressourcen. Im Kern ging es um die Organisation des Arbeitsprozesses, in dem sich alle Faktoren brennpunkthaft bündelten. „Akkordlohnchancen und Kündigungsgefahr bedingen in der kapitalistischen Erwerbsordnung primär die Arbeitswilligkeit", hatte Max Weber in Wirtschaft und Gesellschaft geschrieben. ${ }^{6}$ Gerade diese „Arbeitswilligkeit" war es, die nach dem sukzessiven Rückgang der Arbeitsleistungen, dem Disziplinverfall und der Erosion der betrieblichen Hierarchien zur Debatte stand. Die Hebung der Arbeitsleistung wurde zumindest für Unternehmer und Behörden der Schlüssel für die Überwindung der wirtschaftlichen Nachkriegsschwierigkeiten. Betrachten wir zusammenfassend diese Konstellation für die untersuchten Fallbeispiele.

Für die Unternehmensleitungen sowohl der Farbwerke in Leverkusen als auch der Abteilung Bergbau der VSt. bzw. ihrer Vorgängerunternehmen waren die Zielstellungen in der Nachkriegszeit relativ klar.' Beide Unternehmen hatten bereits vor dem Krieg einen großen Teil ihrer Skalenerträge auf den internationalen Märkten erwirtschaftet, so daß die Rückkehr auf die Weltmärkte und die Wiedergewinnung relevanter Marktanteile in der Sicht der Unternehmensleitungen überlebensnotwendig waren. ${ }^{8}$ Der Zugang zu den internationalen Märkten war trotz der Inflationskonjunktur schwerer geworden als vor dem Krieg. Die Inflationskonjunktur selbst konnte wegen der gesunkenen Leistungsfähigkeit von Anlagen und Belegschaften zudem nicht maximal ausgenutzt werden. Allen Versuchen, die Leistungsfähigkeit der Arbeiterbelegschaften zu steigern, standen Widerstand insbesondere in der prestigebeladenen Arbeitszeitfrage und sich parallel dazu steigernde Verteilungskonflikte im Wege. Steigende Löhne waren aber nur dort akzeptabel, wo sie über den Preis an die Verbraucher weitergegeben werden konnten, was auf den Inlandsmärkten noch funktionieren mochte, zumal sich Staat,

6 Max Weber, Wirtschaft und Gesellschaft, S. 87.

7 Siehe für die Zeit der Weltwirtschaftskrise Zollitsch, Arbeiter zwischen Weltwirtschaftskrise und Nationalsozialismus, S. $108 \mathrm{ff}$.

8 Carl Duisberg, Die Lage der deutschen Wirtschaft. 
Gewerkschaften und Unternehmer in der Kohlenwirtschaft sowohl als Tarifvertragspartner wie als Preissetzungsinstanz trafen. Doch war selbst hier nach der Stabilisierung der Ausweg, höhere Löhne durch Kohlenpreiserhöhungen zu finanzieren, verbaut.

In Leverkusen zeichnete sich recht rasch eine differenzierte Strategie des Werkes zur Lösung der anstehenden sozialen Probleme ab. Zwei Elemente bestimmten in verschiedenen Variationen die Grundinteressen, die vom Direktorium formuliert wurden. Erstens ging es um die Wiederherstellung der betrieblichen und Arbeitsdisziplin und ihre zukünftige Aufrechterhaltung. Zweitens sollte ein rigoroser Sparkurs und eine über Akkordarbeit vermittelte Leistungssteigerung die Wirtschaftlichkeit des Werkes verbessern. Die Werksleitung verfügte dabei über verschiedene Handlungsmöglichkeiten, die mit unterschiedlichen Machtressourcen ausgestattet waren. Zunächst existierte die Möglichkeit einer Optimierung der Arbeitsorganisation und der Effektivierung der Ausnutzung der menschlichen Arbeitskraft über Akkorde, Veränderung der Arbeitsbedingungen und Variation der Arbeitszeit. Diese Möglichkeiten konnten zum Teil autonom, zum Teil nur nach Absprachen mit der betrieblichen Interessenvertretung genutzt werden. Eine weitere Handlungsvariante waren materielle Zugeständnisse in Form von Lohnsteigerungen, Zulagen, angemessenen Akkordsätzen und eine materiell umfangreiche, ausdifferenzierte betriebliche Sozialpolitik. Die Machtressource hier waren materielle Handlungsspielräume, die das Werk wegen seiner guten Liquidität durchweg, selbst noch in der Weltwirtschaftskrise besaß. Jedoch bestand auch hier keine völlige Handlungsautonomie, da einerseits die Löhne tariflich geregelt wurden, andererseits zumindest Teile der betrieblichen Sozialpolitik mitbestimmungs- bzw. mitwirkungspflichtig waren. Drittens konnte eine „rationale“ Belegschaftspolitik betrieben werden, die vor allem über die Einstellungs- und Entlassungspolitik auf Zusammensetzung und Leistungsfähigkeit der Arbeiterschaft Einfluß nahm. Auch hier waren zumindest in wichtigen Fragen die Handlungsmöglichkeiten der Unternehmensleitung über die Mitbestimmungsrechte des Betriebsrates konditioniert. In dieser Begrenzung der Handlungsmöglichkeiten der Werksleitung in Leverkusen wurde die Neudefinition von Eigentums- und Verfügungsrechten durch die Gesetzgebung zur Betriebsverfassung mithin konkret spürbar.

Die Gelsenkirchener Bergwerks AG sowie die anderen Vorgängerunternehmen der späteren Abteilung Bergbau der VSt. verfolgten die gleichen zwei Basisziele wie die Farbenfabriken in Leverkusen. Ihre Handlungsmöglichkeiten und Machtressourcen waren indes unterschiedlich. Die Handlungsvariante materieller $\mathrm{Zu}$ geständnisse, so weit sie sich nicht auf die Lohnhöhe direkt bezog, war sowohl traditionell vernachlässigt worden als auch wegen der nach der Inflation großen Liquiditätsprobleme schwer zu nutzen. Die ohnehin gering entwickelte betriebliche Sozialpolitik verlor in der zweiten Hälfte der zwanziger Jahre weiter an Bedeutung und erreichte erst in der zweiten Hälfte der dreißiger Jahre als nunmehr versteckte Form von Lohnerhöhungen ein etwas größeres Ausmaß. Doch noch im Kontext der Gründung der Chemischen Werke Hüls kam es zu einer Kontroverse zwischen der IG-Zeche Auguste Victoria und der neuen chemischen Fabrik, da die Zeche starke Belegschaftsverluste wegen ihrer im Vergleich zur Chemie 
unterentwickelten betrieblichen Sozialpolitik befürchtete. ${ }^{9}$ Eine „rationale“ Belegschaftspolitik über Einstellungen und Kündigungen wurde von der GBAG nach dem Ende der Inflation betrieben; da es scheinbar allerdings ein Überangebot an Bergleuten gab, das freilich erst die Voraussetzung für eine leistungsorientierte Selektion bot, wurde insbesondere die Nachwuchsförderung vernachlässigt und die seit 1925 wellenförmig erfolgende Abwanderung von Arbeitskräften aus dem Bergbau nicht recht ernst genommen. Erst seit 1926/27 und dem Entstehen eines Mangels an qualifizierten Bergarbeitern nahmen die Bemühungen der Abteilung Bergbau zu, über gezielte Nachwuchsförderung das Angebot an qualifizierten Bergarbeitern zu erhöhen. Doch bereits die Weltwirtschaftskrise entzog dieser Politik wiederum den Boden. Im Bereich der Beschäftigungspolitik blieb daher die leistungsorientierte Selektion das einzig wirksame Mittel der Zechen. Eine Verbesserung der betrieblichen Organisation und Kommunikation erfolgte bei der Abteilung Bergbau nicht; lediglich im Bereich der Verwaltung des Mutterkonzerns, der Vereinigten Stahlwerke, spielte dies eine Rolle. Organisation und Kommunikation erfolgten weiterhin im „bewährten" autoritären Muster. Anders als bei den Farbwerken in Leverkusen wurde die zentrale Handlungsvariante bei der GBAG die bereits geschilderte Rationalisierung. Als technische und betriebswirtschaftliche Straffung von Förderung und Weiterverarbeitung fiel sie in die ausschließliche Handlungskompetenz des Vorstandes. Einer kommunikativen Untermauerung im Betrieb schien sie nicht zu bedürfen. Machtressource war hier die Finanzierbarkeit der Rationalisierung, die einerseits vom Zugang zum internationalen Kapitalmarkt, andererseits von der Liquidität des Unternehmens abhing. Insofern spielten als weiterer Ansatzpunkt Arbeitszeit und Gedingehöhe eine wesentliche Rolle, war doch der Zusammenhang zwischen sinkenden Leistungen, stark zurückgehender Arbeitszeit sowie wachsenden Lohnkosten und Unternehmensliquidität in den Augen der Bergwerksleitungen schlagend. Anders als bei der Rationalisierung aber war in diesem Bereich die Handlungsautonomie der Werksleitungen nach 1918/20 begrenzt. Nicht zuletzt diese Handlungsrestriktion, die sich in wiederkehrenden Arbeitszeitkonflikten äußerte, sowie die extremen Revolutionserfahrungen mit der sich anschließenden Sozialisierungsbewegung begründeten bei den Schachtanlagen der späteren Abteilung Bergbau der VS. eine klare Ablehnung des Weimarer Arbeitsrechtes. Während die Farbenfabriken in Leverkusen das neue Arbeitsrecht akzeptierten und als Mittel betrachteten, die Erreichung der gesetzten Zielstellung unter Umständen sogar zu erleichtern, existierte in der Sicht der Zechenleitungen ein klarer Widerspruch zwischen den betriebswirtschaftlichen Zwängen und den arbeitsrechtlichen Strukturen. Dieser Widerspruch resultierte dabei in dieser Perspektive nicht zuletzt aus der Art und Weise, wie die Belegschaften und Interessenvertretungen das neue Arbeitsrecht auffaßten.

Waren mithin holzschnittartig die Unternehmensleitungen an Kostensenkungen und Wiedergewinnung von (Welt-)marktanteilen interessiert, so ging es den Belegschaften und ihren Vertretungen im wesentlichen ebenfalls um zwei Punkte:

9 Exposé des Direktors von Auguste Victoria, 6. 9. 1940, an den Vorstandsvorsitzenden der I.G. Farben, Schmitz, versandt am 14.9. 1940, ThA RSW 123/02/4b. 
einerseits die Verbesserung ihrer materiellen Lage, andererseits die Reduzierung der Arbeitsbelastungen. Kürzere Arbeitszeiten und höhere Löhne waren ganz eindeutig das primäre Interesse der Belegschaften, denen alle anderen Fragen wie Unfallschutz, Ausbildung oder auch Entwicklung des „Betriebswohls“ nachgeordnet wurden. Alle betrieblichen Entwicklungen wurden letztlich daraufhin befragt, wie weit sie diesen Grundsatzpunkten entsprachen. Kam es in diesen Fragen nicht zu Verbesserungen, so war die jeweils in Frage stehende Belegschaft oder Arbeitergruppe auch nicht an anderweitigen oder Verfahrensverbesserungen interessiert, ja lehnte sie unter Umständen gerade deshalb ab, weil man sich damit „abgespeist" fühlte.

Diese pauschale Beurteilung, wie sie vor allem Gewerbe- und Bergaufsicht zeichnete, war im Kern sicher richtig, doch stellte sich das Bild je nach Branche und Betrieb sehr unterschiedlich dar. Von einheitlichen Arbeiterinteressen auf der Ebene der Betriebe, die zudem einheitlich formuliert wurden, konnte überhaupt nur in Ausnahmefällen die Rede sein. Die Differenzierung der Interessen ergab sich aus zwei Gesichtspunkten. In der Lohnfrage war mit dem Tarifsystem im Prinzip klar, daß die Ebene der grundsätzlichen Lohnfindung, bei der noch am ehesten einheitliche Interessen der Beschäftigten existierten, sich tendenziell vom Betrieb wegbewegte. Spätestens nach der Stabilisierung der Mark war der Betrieb nicht mehr der Ort der Lohnauseinandersetzungen und dies blieb - von Ausnahmen abgesehen - trotz aller Arbeitgeberversuche zur Tarifflexibilisierung auch in der Weltwirtschaftskrise so. Gleichwohl gab es betriebliche Lohnbewegungen, die in der Regel auf der Basis fester Tarifzeitlöhne mit der Ausgestaltung der Leistungslohnsysteme verbunden waren. Die Akkordarbeit war nach der Revolution zwar kurzfristig generell abgelehnt worden, danach aber griff sie wieder sehr schnell und in der Regel mit Zustimmung relevanter Belegschaftsteile Platz, denen häufig kaum etwas anderes übrigblieb, wollten sie ihre Lohnsätze deutlich erhöhen. Die Akkorde in der Chemie wie die Gedinge im Bergbau betrafen allerdings die Belegschaften nicht gleichmäßig, so daß zwangsläufig eine Interessendifferenzierung auftrat. Zwar wurde im Bergbau durchgängig im Akkord gearbeitet, doch auch hier war die Akkordaushandlung entsprechend der Abbaulage und der einzelnen Kameradschaften differenziert. In den Betrieben fehlte daher in der Regel der Grad der Vereinheitlichung der Belegschaften, der dauerhaftes solidarisches Handeln ermöglicht hätte. Durch eine bewußt differenzierende Lohnpolitik, die sich die je nach Arbeitsplatz unterschiedlichen Arbeitsinhalte und Leistungsanforderungen bewußt zu Nutze machte, wurde dieses Phänomen namentlich in der chemischen Industrie weiter verstärkt. Eine Anpassung der Lohnsysteme an die Struktur der Arbeitsprozesse hatte daher fast zwangsläufig Differenzierungsfolgen, was von vielen Belegschaftsangehörigen, die in ihren Lohnforderungen in der Regel von der eigenen Arbeitsplatzwirklichkeit ausgingen, selbst allerdings nachhaltig unterstützt wurde.

Die andere Seite des Problems bestand darin, daß die Belegschaften sozial nicht homogen waren und sich mit dieser Heterogenität jenseits der Lohninteressen auch andere Zielsetzungen verbanden. Hier bestand ein gravierender Unterschied zwischen chemischer Industrie und Bergbau, da in letzterem der soziale Aufstieg viel stärker limitiert war als in der chemischen Industrie. Dort bestand eine zu- 
mindest statistisch größere Wahrscheinlichkeit gegebenenfalls Vorarbeiter oder nach entsprechenden Kursen im Werkstattbereich - auch Meister zu werden ${ }^{10}$, während im Bergbau diese Aufstiegsmöglichkeit für das Gros der Belegschaft faktisch ausgeschlossen war. Von jenen Arbeitern, die in Leverkusen vor 1914 länger als zehn Jahre beschäftigt waren, hatten immerhin $35 \%$ den „Aufstieg“ zu Vorarbeitern (21\%), Aufsehern (13\%) und Handwerksmeistern (1\%) geschafft. ${ }^{11}$ Stärker aber als diese Differenzierung wirkte in der Chemie noch das Berufsbewußtsein der ausgebildeten "Handwerker" gegenüber den ungelernten „Massenarbeitern", deren innerbetriebliche Differenzierung sich auch außerhalb des Betriebes fortsetzte, wo - wiederum anders als im Bergbau - keine soziale Infra- und Kommunikationsstruktur existierte, die der betrieblichen Differenzierung außerbetrieblich entgegengewirkt hätte. Zwar wohnte der überwiegende Teil der Leverkusener Belegschaft in der Nähe des Werkes, das Sozialleben der verschiedenen Gemeinden Leverkusens war aber gerade deshalb weitgehend durch das Werk und seine umfängliche Sozialpolitik dominiert. Eine Vielzahl von untereinander wiederum sozial abgegrenzten Vereinen, die werksseitig unterstützt wurden, hielten das öffentliche und Freizeitleben besetzt, und selbst wenn „klassenbewußte“ Arbeiter diese nicht frequentierten, so verblieb doch ein nicht unbedeutender Teil der Belegschaft in diesen Strukturen verhaftet, wobei dieser Anteil mit dauernder Werkszugehörigkeit wuchs, also insbesondere die Stammbelegschaft von der „Bayer-Familie“ integriert wurde. Die spezifische Form der Personalrekrutierung, die Bayer vor dem Krieg mit Erfolg eingeführt hatte, nämlich Werksangehörigen Werbeprämien für vermittelte Arbeitskräfte zu zahlen, die eine bestimmte Frist im Werk blieben, wirkte sich hier doppelt aus, da in der Regel die Werbung durch bereits integrierte Stammarbeiter erfolgte, die ihrerseits sozial disziplinierend auf ihre "Neuwerbungen“ einwirkten. ${ }^{12}$ Ein typisches Berufsbewußtsein existierte im übrigen bei einer großen Zahl der ungelernten Chemiearbeiter selbst in den fünfziger und sechziger Jahren nicht, und entwickelte sich, wenn überhaupt, so erst nach längerer Werkszugehörigkeit. ${ }^{13}$ Dieses Berufsbewußtsein bzw. seine Ansätze aber griff das Werk durch seine Berufsvereine gezielt auf, die zwar nach der Revolution 1918 in die Defensive gerieten , aber nicht völlig verschwanden. Anders als beim Bergbau blieb das Berufsbewußtsein des Chemiearbeiters an einen bestimmten, dauerhaft besetzten Arbeitsplatz gebunden und mußte bei Fluktuation schnell verlorengehen bzw. konnte gar nicht erst entstehen. Chemiearbeit war eben nicht eindeutig definiert; ein Bewußtsein konnte erst mit der Tätigkeit selbst entstehen, nicht aufgrund abstrakter Berufsbilder, die gar nicht existierten. Dies galt vor allem für die ungelernten Chemiearbeiter; die „Handwerker" verfügten über ein ausgesprochenes, über die Qualifikation vermitteltes Bewußtsein, das sie aber nicht mit der Chemie verband. Viele Leverkusener „Handwerker" wechselten in die Kölner oder die Industrie des Ruhrgebietes, wenn die

$10 \mathrm{Im}$ Bereich der chemischen Produktion konnte man im übrigen bis zum Werkmeister ohne jede formale Qualifikation aufsteigen. „Ungelernte Werkmeister“ dürften dabei zweifellos eine Spezialität der chemischen Industrie gewesen sein, Lindemann, Die Arbeitsverhältnisse, S. 21.

11 Curt Duisberg, Die Arbeiterschaft der chemischen Industrie, S. 41.

12 Siehe für die Vorkriegszeit R. Grabendörfer, Die Arbeiterverhältnisse in Leverkusen, BAL 212/1.

13 Vgl. Fürstenberg, Der Chemiearbeiter. 
Lohnunterschiede dies attraktiv machten, ohne offensichtlich ihrem, häufig wegen seiner spezifischen Belastungen wohl eher abgelehnten Chemiearbeitsplatz auch nur eine Träne nachzuweinen. Strenggenommen konnte es also auch hier wie bei den ungelernten Arbeitern ein spontanes Chemiebewußtsein nicht geben. Was es geben konnte und was es gab, war ein durch die Dauer der Betriebszugehörigkeit entscheidend konditioniertes Bayer-Bewußtsein, in das Elemente einer Art chemischen Berufsbewußtseins einflossen. Das Berufsbewußtsein aber als eine Identitätsform, die auch gegen den Arbeitgeber und außerhalb der Arbeit Berufsstolz und Selbstbewußtsein generierte, war in der Chemie nur schwer vorstellbar, zumal in der Öffentlichkeit die chemischen Fabriken („Giftküche“) meist mit noch schlimmeren Pejorativen als die Zechen („Kohlenpott“) bedacht wurden. ${ }^{14}$

Stellte Leverkusen daher in gewisser Weise eine vom Werk her strukturierte und in ihren Kommunikations- und Bewußtseinsformen zumindest mitbestimmte Gemeinde dar, so galt dies für den Bergbau und seine Kolonien kaum, da es abgesehen von Deputatkohlen und einem nach 1918 allerdings geringen Werkswohnungsbau kaum Versuche gab, die Bergleute auch jenseits des Arbeitsprozesses in ihren Arbeitszusammenhang sozial zu integrieren. Die Bergarbeiterkolonie blieb daher zwar vom Bergbau und seiner Arbeit bestimmt, auf den sie sich kommunikativ und sozial bezog. Sie war indes weit autonomer als die Wohngemeinschaften in Küppersteg oder Wiesdorf. Dies hing auch mit dem ausgeprägten Berufsbewußtsein und Berufsstolz der Bergleute zusammen, der nicht an einen bestimmten Arbeitsplatz oder Arbeitgeber gekoppelt war. Die geringere soziale Differenzierung auf der Zeche fand damit ein passendes Gegenstück in der Kolonie. ${ }^{15}$ Geringere soziale Differenzierung der Arbeiterschaft und starkes, zudem moralisch codiertes Berufsbewußtsein waren höchstwahrscheinlich Gründe, die die hohe Mobilisierbarkeit und die Eskalationsbereitschaft der Bergarbeiterschaft mit ermöglichten. Dieses Berufsbewußtsein wurde allerdings spätestens seit Beginn der Strukturkrise 1924 einem tiefgreifenden Erosionsprozeß unterzogen, da der Bergbau nicht mehr die erwünschte soziale Sicherheit bot. In Wellen 1924/25 und 1927/28 begannen Abwanderungsprozesse aus dem Bergbau, die in der Regel die jüngeren und qualifizierteren Arbeitskräfte erfaßten, da diese in den Nachbarindustrien leichter Fuß fassen konnten. Die Strukturkrise zerstörte damit das soziale Gefüge, vor allem das Selbstbild des Bergbaus weitgehend und nahm den Bergarbeitern einen Teil ihres bisherigen Selbstbewußtseins. Der Rückgang der Kampfbereitschaft nach 1924 dürfte auch mit dieser Krisenerfahrung eng verbunden gewesen sein.

Wirkten betriebliche Lohnkonflikte und arbeitsplatzbezogenes Karriereverhalten im betrieblichen Kontext daher unter Umständen eher differenzierend und erschwerend für das gemeinsame Handeln der Belegschaften, zumal dann, wenn ein vereinheitlichendes Berufsbewußtsein nicht bestand, so spielte die Arbeitszeit-

14 Zum Bewußtsein von Chemiearbeitern gibt es keine historischen Studien. Hinweise finden sich bei Kern, Schumann, Das Ende der Arbeitsteilung.

15 Siehe die umfassende Diskussion von Faktoren, die die besondere Militanz des Bergarbeitermilieus beeinflußten, bei Hartewig, Das unberechenbare Jahrzehnt, S. $244 \mathrm{ff}$, die freilich die Interaktivität sozialer Prozesse ein wenig vernachlässigt. Insbesondere die Betrachtungen zur Märzrevolution sind daher wenig komplex. Vgl. als ein Beispiel für bergmännisches Leben auch Zimmermann, Schachtanlage und Zechenkolonie. 
frage zumindest auf den ersten Blick eine andere Rolle, da sie in ihrer Bedeutung eher vereinheitlichend als differenzierend wirkte, auch wenn in der chemischen Industrie für die einzelnen Arbeiterkategorien und Tätigkeiten unterschiedliche Zeitsysteme und Arbeitszeiten in Geltung waren. Die Bedeutung der Arbeitszeitfrage resultierte aus zwei Quellen. Einerseits handelte es sich um eine traditionelle, symbolisch außerordentlich stark aufgeladene Forderung der Arbeiterbewegung. Die Durchsetzung des Achtstundentages 1918 wurde in vielen Augen gleichsam zum entscheidenden Signum der Revolution. Zum anderen bündelten sich in der Arbeitszeitfrage am sichtbarsten die Wünsche und Forderungen zur Verbesserung der Arbeitsbedingungen. Durchgreifende Erfolge erwarteten die Belegschaften in der Regel weniger von einem verbesserten Unfallschutz als vielmehr von kürzeren Arbeitszeiten bei vollem Lohnausgleich, die als Kompensation für die schwere Arbeit begriffen wurden. Insbesondere die Krise der Nahrungsmittelversorgung seit 1916 schien eine verkürzte Arbeitszeit nachgerade zu erzwingen. Diese Forderung wurde sowohl im Bergbau wie in der chemischen Industrie von allen Belegschaftsteilen durchweg vertreten, auch wenn die einzelnen Arbeitszeitsysteme durchaus unterschiedlich waren. Im Bergbau variierten sie vor allem zwischen den Unter- und Übertagearbeitern, wobei Unter Tage die Wechselschicht mit Arbeitszeiten zwischen sieben (nach 1918) und neun (vor 1918) Stunden vorherrschte, über Tage im Zweischichtsystem mit zehn bis zwölfstündiger Arbeitszeit vor 1914, danach die Wechselschicht mit acht- und schließlich mit neunstündiger Regelarbeitszeit vorherrschte. In der Chemie arbeiteten die Handwerker in zehn- bis zwölfstundigen Doppelschichten, bei den Anlagenbedienern variierten die Arbeitszeiten je nach Struktur des Arbeitsprozesses. Zwischen 1918 und 1923 dominierte die achtstündige Regelschicht, danach ging Bayer zur neunstündigen Regelschicht über. Als Problem stellte sich nach 1918 nicht nur die Regelarbeitszeit, die zu verlängern die Werksleitungen sich regelmäßig und im Bergbau nachdrücklich bemühten, sondern mehr noch die Überstundenfrage. Die Werksleitungen sowohl in Leverkusen wie im Bergbau bemühten sich, das Arbeitsvolumen über Überstunden flexibel den Marktverhältnissen anzupassen, was von den Belegschaften naheliegenderweise zunächst als Versuch, den Achtstundentag zu durchbrechen, abgelehnt wurde, später zum Ansatz zumindest für erhebliche Lohnforderungen im Sinne von Überstundenzuschlägen genutzt wurde. Arbeitszeit- und Überstundenregelungen, auch wenn sie differenziert im Betrieb realisiert wurden, waren gleichwohl jeweils nur generell zu treffen, so daß sich hierüber eine Vereinheitlichung der Interessen der Belegschaft auf einen bestimmten Punkt hin einstellte. Symbolik, reale Bedeutung der Arbeitszeitverkürzung, Lohn- und Zulagenforderungen traten hier in enger Kombination auf und bedingten durch die Vereinheitlichung eine Verstärkung der Machtressourcen der Belegschaften.

Es ist daher kein Wunder, daß die bedeutendsten Konflikte sowohl im Bergbau als auch in der chemischen Industrie in der Arbeitszeitfrage geführt wurden. Dabei ergaben sich drei unterschiedliche Phasen. Während in den Jahren zwischen 1918 und 1923 die politische Bedeutung des Achtstundentages im Vordergrund stand, wurde die Mehrarbeit zwischen 1924 und 1929 vor allem zu einer Frage des Lohneinkommens. Mehrarbeit wurde nach 1924 von den Belegschaften nur noch 
zu einem geringer werdenden Teil aus politischen Gründen abgelehnt; es dominierte jetzt vielmehr das Interesse an einem möglichst hohen Mehrverdienst. Hierbei dürfte die Erfahrung eine wesentliche Rolle gespielt haben, daß fast alle Arbeitszeitkämpfe in der frühen Nachkriegszeit entweder verlorengegangen waren oder nur mit politischer Unterstützung halbwegs gewonnen werden konnten. Vor allem aber spielte nach der Inflation das niedrige Reallohnniveau die Rolle des Katalysators, der einer politischen Betrachtung der Arbeitszeitfrage den Boden entzog. Betriebsräte und Gewerkschaften, die den Achtstundentag aus politischen und volkswirtschaftlich-strukturellen Gründen verteidigen wollten, gerieten auf diese Weise in einen Doppelkonflikt mit Unternehmensleitungen und relevanten Belegschaftsteilen. In der Weltwirtschaftskrise schließlich brachte das Arbeitsmarktrisiko Arbeitszeitkämpfe von Seiten der Belegschaften zum Erliegen. Während in der Chemie die Arbeitszeitverkürzungen von den Betriebsräten begrüßt wurden, da sie die Aufrechterhaltung eines hohen Beschäftigungsniveaus garantierten, wurden Feierschichten im Bergbau von den Betriebsräten und den noch angelegten Bergleuten bekämpft, da das Beschäftigungsrisiko hierdurch nicht sank, die Arbeitseinkommen allerdings stark zurückgingen. Das Verhalten der Belegschaften läßt sich ökonomisch vielleicht am besten über die Opportunitätskosten des Achtstundentages begreifen. So lange der Freizeit- und Erholungsgewinn durch kürzere Arbeitszeit nicht durch Einkommensverluste mehr als ausgeglichen wurde, dominierte eine letztlich politische Sichtweise der Arbeitszeitfrage. Mit dem Steigen der Opportunitätskosten einer kurzen Arbeitszeit nach dem Ende der Inflation, änderten sich ihre Beurteilungskriterien und der mögliche Einkommenszuwachs wog schwerer als der Freizeitverlust.

Die Rolle der Betriebsräte in der Arbeitszeitfrage wandelte sich entsprechend der Belegschaftshaltung bzw. des Wandels der Opportunitätskosten in der Arbeitszeitfrage. Wurde die Arbeitszeitmitbestimmung zunächst noch als Mittel zur Aufrechterhaltung des Achtstundentages begriffen, so ging es ab 1924 stärker um die Sicherung und Steigerung der Einkommen von Chemie- und Bergarbeitern. Damit wandelte sich auch der betriebliche Interessenvertretungspragmatismus. Stand dieser bis 1923 cum grano salis noch in Einklang mit zentralen gewerkschaftlichen Forderungen, so bildete sich nach 1924 ein neues Muster betrieblicher Interessenvertretung heraus, das nicht mehr ohne weiteres von der gewerkschaftlichen Programmatik ausgehen konnte. Das verbreitete Mißbehagen namentlich der sozialistischen Gewerkschaftsprogrammatiker gegenüber den betrieblichen Interessenvertretungen reflektierte einen Zusammenhang, in dem das Verhalten der Belegschaften sich als egoistisch und die entsprechenden Handlungsimpulse für die Betriebsräte sich als syndikalistisch zu erweisen schienen. Betriebsräte, die sich weiterhin primär an der Gewerkschaftsprogrammatik orientierten, gerieten in erhebliche Loyalitätskonflikte. Die Weltwirtschaftskrise „individualisierte" das Verhalten der Arbeiterschaft noch weiter; selbst die Interessenvertetung über die Betriebsräte wurde jetzt als wenig effektiv wahrgenommen, da auch deren Tätigkeit die Beschäftigungs- und Einkommensrisiken nicht mehr verringern konnte. Es kam daher fast zwangsläufig zu dem Paradoxon von zwar wahlradikalen, aber nicht mehr handlungsbereiten Belegschaften. Die Belegschaften begriffen ihre eigene Lage als bedauernswert und machten hierfür das politi- 
sche und soziale System verantwortlich, besaßen aber keine eigenen Handlungsspielräume, um Änderungen des Systems durchsetzen zu können. Ihre Proteste wurden daher symbolisch.

Wie weit die Politik bei der Interessenartikulation und -durchsetzung der Belegschaften eine Rolle spielte, hing neben den Opportunitätskosten des Belegschaftshandelns nicht zuletzt von den bestehenden betrieblichen Kommunikations- und Handlungsmöglichkeiten ab. Sicher ist der unterschiedliche Grad der Politisierung der Belegschaften im Bergbau und der chemischen Industrie. Zwar votierten in beiden Branchen sowohl bis 1924 als auch nach 1930 relevante Belegschaftsteile für radikale Gruppierungen bei den Betriebsratswahlen, jedoch spielten politische Forderungen im betrieblichen Alltag in der chemischen Industrie eine eher nachgeordnete Rolle. Es war offensichtlich, daß eine Politisierung der Belegschaft mit dem Versagen innerbetrieblicher Kommunikation oder mit der Vorstellung, auf friedlichem Wege sei letztlich nichts zu erreichen, eng korrespondierte. Die Politisierung war dann ein Ausweg aus der betrieblichen Blockade, der aber nicht automatisch beschritten wurde, sondern der Artikulationshilfe bedurfte bzw. den Ansatzpunkt politischer Gruppen bildete. Bei Bayer Leverkusen war die Politisierung stets das Zusammenspiel von Kommunikationsversagen und einschlägiger Agitation, wobei sie sich vom Bergbau nur deshalb unterschied, weil sich an dessen Politisierung Staat und Parteien gleichsam qua Amt beteiligten, die „Agitation“, die die betrieblich-sozialen zu politischen Grundsatzfragen erklärte, im Kontext der Sozialsierungsdebatte also gleichsam institutionalisiert wurde. Im Bergbau profitierte die Politisierung der sozialen Konflikte zudem von weiteren Faktoren wie der Häufung der Schachtanlagen im Ruhrgebiet und der großen volkswirtschaftlichen Bedeutung der Branche, wodurch sie wegen der Produktionseinbrüche nach 1918 zwangsläufig zum Gegenstand der Politik wurde. Anders als im Rheinland verhinderte hier auch keine Besatzungsmacht die Eskalation betrieblicher Konflikte. Die Bergleute konnten daher die Politisierung ihrer Forderungen nicht nur ungehindert vollziehen, sie wurden durch die öffentlichen Debatten hierzu nachgerade aufgefordert. Dadurch kam aber in die betrieblichen Konflikte zumindest in manchen Zechen des Ruhrgebietes eine Erbitterung, die man am Rhein nur ganz kurzfristig kannte. Gerade letzteres aber war es, was der Politisierung ihre Bedeutung verlieh. Politisierte Konflikte ließen sich im Betrieb nicht mehr regulieren, da sie auf die Unterdrückung der Gegenseite abzielten, nicht auf die Konfliktkooperation. So sehr die Politisierung daher Ausdruck versagender Kooperation war, so sehr bedingte sie auch das Versagen der Kooperation. Es mußte im Einzelfall vom Geschick der Beteiligten abhängen, ob dieser Zirkel durchbrochen wurde.

Gerade hierfür aber waren die Voraussetzungen im Bergbau denkbar schlecht. Hatten bis zum Ende der Inflation vor allem die Bergarbeitergewerkschaften die Frage der Zukunft des Bergbaus fast notgedrungen politisiert, um die „direkten Aktionen“ der Bergarbeiterschaft noch einigermaßen im Griff halten zu können, und zumindest verbal auch wichtige Ergebnisse erreicht, so begann danach eine umgekehrte Politisierung. Spätestens ab 1926 und dem Hinaufschnellen der „sozialversicherungsbedingten“ Förderausfälle auf den Zechen des Ruhrgebietes und einer gleichzeitig deutlich steigenden Abgabenlast (vor allem des sog. Knapp- 
schaftsgefälles) begann eine Politisierung des Bergbaus von der Zechenseite her. Da man ganz offensichtlich die innerbetrieblichen Sozialbeziehungen nicht in den Griff bekam, verlangte der Bergbau von der Politik Maßnahmen, die ihm eine repressive Disziplinierung der angelegten Arbeiterschaft erlaubt hätten. Aus der Sicht der Belegschaften ist festzuhalten, daß eine "Politisierung“ der Konflikte nicht den Anfang der Auseinandersetzungen bildete, sondern regelmäßig erst dann eintrat, wenn der Verhandlungsweg erfolglos schien bzw. die Verhandlungen sich derart in die Länge zogen, daß ein weiteres Zuwarten nicht mehr hinnehmbar schien. Die Politisierung korrespondierte daher eng mit dem Versagen der geregelten Konfliktaustragung bzw. mit der Enttäuschung der Belegschaften über die Ergebnisse geregelter Konfliktbewältigung. Sie schuf indes Räume für die Arbeit von Gewerkschaften und politischen Gruppen, die eine Tendenz hatte, sich zu verstetigen. Auf diese Weise wurde die ohnehin vorhandene soziale und materielle Differenzierung der Arbeiterschaft durch eine politische Differenzierung ergänzt, die den Betriebsräten das Handeln nur weiter erschwerte, da sie sich auf keine einigermaßen einheitlichen Willensbildungsprozesse der Belegschaften mehr einstellen konnten.

Ließen sich also durchaus bestimmte, wenngleich auch nach Beschäftigten- und Qualifikationsgruppen variierende Basisinteressen abhängig beschäftigter Lohnarbeiter bestimmen, so hing deren konkrete Formulierung von den Bedingungen $a b$, unter denen sie innerhalb und außerhalb der Betriebe kommuniziert werden konnten und mußten. Auch die Art und Weise ihrer Durchsetzung korrespondierte eng mit der Art und Weise, wie sie formuliert wurden. Daher war die Werkstatt- oder Arbeitsgruppe zunächst jener soziale Rahmen, in dem Forderungen aufgestellt und eingeklagt wurden. Erst wenn diese Form versagte oder zumindest entwertet wurde, kamen übergeordnete Ebenen ins Spiel, die aber zugleich zu übergeordneten Forderungsformulierungen gezwungen waren. Die eigentliche Ausgangsebene (Werkstatt, Arbeitsgruppe) blieb für den einzelnen Beschäftigten aber jener Raum, in dem er kommunizieren und daher auch die Entwicklung der übergeordneten Ebene debattieren konnte. Die Bereitschaft der Einordnung in größere Bewegungen war daher widersprüchlich. Sie konnte zur Durchsetzung von Interessen förderlich sein, freilich von Interessen, die auf der Werkstatt- oder Gruppenebene formuliert worden waren. Letztlich war damit das Arbeiterverhalten nicht einheitlich, weder in der Chemie noch im Bergbau, auch wenn bei letzterem hierfür die Voraussetzungen günstiger waren. Die Revolution machte etwas an sich sehr Unwahrscheinliches schlagartig möglich: das gemeinsame Auftreten von im Prinzip nicht homogenen Arbeitergruppen.

Den Belegschaften insgesamt blieb als kollektive Machtressource lediglich der Streik, doch war dieser nur unter erheblichen Risiken einsatzfähig, solange nicht die Tarifgewerkschaften mitspielten. Deren Rolle in Leverkusen und bei der GBAG war ohnehin eine eher schwache. Zwar gingen gegen Kriegsende und in der Inflation die Organisationsziffern nach oben, doch die schweren Streik- und Aussperrungskämpfe namentlich der Jahre 1920/21, die ohne bzw. gegen die Tarifgewerkschaften durchgeführt und verloren wurden, zerstörten die tarifgewerkschaftliche Basis in den Betrieben. Hyperinflation und Art und Weise der Anpassungskrise nahmen Berg- und Fabrikarbeiterverband schließlich ein weiteres 
Stück an Legitimität. Mitgliedschaft in einer Tarifgewerkschaft war bei Bayer und auf den Schachtanlagen der späteren Abteilung Bergbau der VSt. nach 1924 eher die Ausnahme als die Regel. ${ }^{16}$ Alternativ zu gewerkschaftlichen Kampfmitteln existierte, solange es keine funktionierende Betriebsvertretung gab, lediglich spontanes Konfliktverhalten am Arbeitsplatz, wo die Machtasymmetrie für den einzelnen Arbeiter sehr rasch und deutlich zu spüren war. Die Hoffnung auf die betrieblichen Interessenvertretungen und ihre Arbeit resultierte daher aus der Situation, selbst legitim nur außerordentlich eingeschränkt handlungsfähig zu sein. Andererseits aber war wiederum ein Teil der artikulierten Interessen im Betrieb ohnehin nicht verallgemeinerungsfähig, so daß hier die Betriebsvertretung zwangsläufig in die Rolle der Vertretungsmacht geriet.

Die Stellung der Betriebsvertretung war daher ausgesprochen prekär. Von Seiten der Belegschaften mit hohen, aber keineswegs einheitlichen Erwartungen belastet, waren sie einerseits gesetzlichen Restriktionen unterworfen und standen andererseits einem unter Umständen kooperationsunwilligen Arbeitgeber gegenüber. Die Belegschaftserwartungen waren aber nur zum Teil explizit, nur zum Teil verallgemeinerungsfähig und frönten häufig einem gewissen „Gruppenegoismus". Die formalen Machtressourcen der Belegschaft waren gering und konnten auch faktisch nur in jenen eher seltenen günstigen Fällen voll mobilisiert werden, in denen die Konfliktgegenstände eine Vereinheitlichung der Belegschaftsinteressen zuließen. Einerseits von der Zustimmung der Belegschaften abhängig, konnten sich die Betriebsräte andererseits kaum dauerhaft und immer auf sie stützen. Die Anlehnung an die Tarifgewerkschaften bot hier einen nicht ganz ungefährlichen Ausweg, da hierdurch ja gerade die Belegschaftszufriedenheit nicht unbedingt gesteigert werden konnte. Es kam daher letztlich alles darauf an, wie sich die jeweiligen Akteure je konkret verhielten. Handlungssicherheit existierte im Grunde nicht.

\section{Konfliktverläufe 1916 bis 1934}

Auf die krisenhafte Verringerung der Leistungsfähigkeit ihrer Belegschaften reagierten nach 1916 sowohl die Farbwerke in Leverkusen als auch die Vorgängerunternehmen der Abteilung Bergbau der VSt. mit einer differenzierten Strategie der Lohnerhöhung, die an das betriebliche Leistungsverhalten gekoppelt war. Die Belegschaftsproteste richteten sich vor dem Hintergrund von Lebensmittelknappheit und Teuerung gegen die zu langen Arbeitszeiten und die zu geringen Lohnanpassungen. Damit waren zwei im Prinzip gegensätzliche, aber doch noch kompromißfähige Positionsbestimmungen auf dem Tisch. Anders als während der

16 Diesen hochinteressanten Fragen konnte in der vorliegenden Arbeit nicht weiter nachgegangen werden, da diese Entwicklung mit der Gesamtpolitik der Gewerkschaften und der Funktionsweise und den Erfolgen des Tarifsystems zusammenhing. Hier lagen ja auch die eigentlichen Gründe für Engagement und Mitgliedschaft in einer Gewerkschaft. Zahlen zur Mitgliedschaft allgemein in: Jahrbuch des Verbandes der Fabrikarbeiter Deutschlands, Ifd., Jahrbuch des Verbandes der Bergarbeiter, Ifd. Organisationsquoten für den Ruhrbergbau bei Zollitsch, Arbeiter zwischen Weltwirtschaftskrise und Nationalsozialismus, S. 190. Zur Chemie vgl. Herfarth, Strukturwandlungen. Des weiteren Uta Stolle, Arbeiterpolitik im Betricb. 
Vorkriegszeit konnten sich die Werksleitungen nach dem Inkrafttreten des Hilfsdienstgesetzes nun aber über die Position ihrer Belegschaften nicht mehr hinwegsetzen, sondern mußten diese mit den Arbeiterausschüssen verhandeln, zumal diesen über ihre Ausschüsse nun auch der Weg zur paritätischen besetzten Schiedsstelle möglich war. Damit geriet die traditionelle Lohn- und Arbeitspolitik der Werksleitungen unter Anpassungsdruck, wollte man einen permanenten, konfliktbesetzten und tendenziell aus dem Betrieb herausführenden Kleinkrieg vermeiden. Anders ausgedrückt, erhöhten die Konsequenzen des Hilfsdienstgesetzes für die Werksleitungen die Interaktionskosten mit ihren Belegschaften in einer Weise, die Modifikationen in den bisherigen Verhaltensweisen verlangte. Während die Werksleitung in Leverkusen auf diesen veränderten Konfliktalltag dadurch reagierte, daß sie gleichzeitig intern ihre lohn- und arbeitspolitischen Strukturen revidierte, extern auf eine Aufhebung des Hilfsdienstgesetzes drang, und gegenüber der Belegschaft Entgegenkommen im Einzelnen bei Aufrechterhaltung ihrer individuellen Lohnpolitik im allgemeinen zeigte, also ihr Verhalten differenzierte, setzten die Zechenleitungen - in Übereinstimmung mit den anderen Großindustrien des Westens - extern auf eine Bekämpfung der Gewerkschaften und eine Beseitigung des Hilfsdienstgesetzes und verfolgten gegenüber den Belegschaften die Politik der leistungsorientierten Lohnerhöhungen weiter. Eine interne Ausdifferenzierung ihrer Arbeits- und Lohnpolitik unterblieb, auch wenn sich ähnlich wie in der Chemie de facto eine Kryptotarifierung der Löhne durchgesetzt haben dürfte. Anders als in Leverkusen, wo es sich um ein einzelnes Werk handelte, brachte diese Verweigerung im Ruhrbergbau nach und nach vereinheitlichende Tendenzen in das Verhalten der Belegschaften und ihrer Gewerkschaften, denen klar schien, daß eine Änderung der Kommunikations- und Verhandlungsstrukturen nicht freiwillig, sondern nur durch politischen Druck erreichbar sein würde. Da die Bergarbeiterproteste und die Bereitschaft der Belegschaften, angesichts der geringen Kompromißbereitschaft der Unternehmensleitungen zu Streikmaßnahmen zu greifen, zu Unruhen und Förderausfällen im Revier führten, griff in die industriellen Beziehungen des Bergbaus der Staat bzw. die zuständige Militärbehörde in der Regel nach Aufforderung durch die Gewerkschaften, die während des Krieges Kampfmaßnahmen vermeiden wollten, direkt ein. Es entstand eine Art indirektes Tarifsystem, insofern die Militärbehörde die Gewerkschaftsforderungen an den Zechenverband mit der Aufforderung weitergab, diese Wünsche wohlwollend aufzunehmen. Auf diese Weise wurde bereits in der zweiten Kriegshälfte das Versagen der betrieblichen und Branchenkooperation gleichsam politisch ausgeglichen, wobei es freilich zunächst jeweils der Drohung der Bergarbeiterschaft mit direkten Aktionen bedurfte, bevor die Militärbehörde im genannten Sinne tätig wurde. Die Kompromißbereitschaft der Zechenbesitzer wurde überdies bereits während des Krieges mit Preiszugeständnissen erkauft; zu wirklichen materiellen Zugeständnissen zwang sie also niemand.

Der Ausbruch der Novemberrevolution brachte für die Farbwerke in Leverkusen daher vor allem den Verzicht auf die politische Hoffnung, man könne den arbeits- und lohnpolitischen Rückweg in das Kaiserreich antreten. Da allerdings die interne Anpassung der lohn- und arbeitspolitischen Strukturen weit fortgeschritten war, brachte die Tarifierung der Löhne und die Durchsetzung des Acht- 
stundentages für das Werk keine strukturellen Neuerungen. Auch die möglichst enge Kooperation mit dem Arbeiterausschuß war zumindest nicht strukturell an weitreichende Änderungen gebunden, so daß sich der "revolutionäre Prozeß“ in Leverkusen auf einen kurzen Zeitraum beschränkte, in dem die Werksleitung durch antizipatives Konfliktverhalten ihre Handlungsmöglichkeiten offenhalten konnte. In Leverkusen war daher die „Revolution“ im Betrieb bereits bewältigt, als sie schließlich auch auf der Ebene der Organisationen ihren Abschluß fand. Da sich das Tarifsystem auf der Branchenebene zudem relativ schnell einspielte und die jeweiligen Tarifkompromisse rasch und ohne staatliche Intervention erzielt wurden, entfiel auch ein großer Teil der Konflikte, die ansonsten nach einer betrieblichen Lösung gedrängt hätten. Im Bergbau war die Situation vollständig anders. Auf den Zechen herrschte im wesentlichen während der Revolution noch Ruhe; der Ausgleich zwischen den Organisationen im November 1918 wurde durch keinen gleichlaufenden betrieblichen Prozeß abgestützt, auch wenn sich die Arbeiterausschüsse häufig spontan neubildeten. Als sich die in den Augen der Belegschaften unzureichenden Ergebnisse der „Revolution“ auf Betriebsebene zeigten, exisitierte hier im Gegensatz zu Leverkusen keine kommunikative Infrastruktur, um eine vergleichsweise reibungslose Transformierung des Tarifsystems auf die betrieblichen Verhältnisse zu ermöglichen. Im Gegenteil dominierte weiterhin die reine Kommunikation in der Linie, die auch die Besprechungen zwischen $\mathrm{Ze}$ chenverwaltungen und Arbeiterausschüssen bestimmte. Der Gedanke, daß Emil Kirdorf sich mit den Arbeitervertretern an einen Tisch gesetzt hätte, war wohl auch wegen der unterschiedlichen Persönlichkeiten von Duisberg und Kirdorf ausgeschlossen, vor allem aber hätte ein derartiges Verfahren im Bergbau gegen die gesamte Kommunikationstradition durchgesetzt werden müssen, wozu sich der Vorstand des Unternehmens nicht aufraffen konnte. So waren auf den Zechen diejenigen, welche für Leistungsstand und Gedingeabrechnung verantwortlich waren, zugleich auch die Ansprechpartner der Arbeiterausschüsse. Daß bei offenen Konflikten im ohnehin stark von autoritären Verhaltensmustern geprägten Bergbau eine Konflikteskalation nahe lag, war daher mehr als wahrscheinlich. Die sich hinziehenden Verhandlungen zwischen Zechenverband und Gewerkschaften sowie die zunächst ausbleibende Tarifierung der Löhne erhöhten den Problemdruck auf den Schachtanlagen, für den aber gerade keine geeigneten Kommunikationsstrukturen existierten. Es war daher kein Wunder, daß sich die ersten massiven Konflikte im Kontext der neuen Arbeitszeitregelungen ergaben. Zwar war der Achtstundentag in Tarifverhandlungen vereinbart worden, seine betriebsspezifische Umsetzung erfolgte jedoch durch die Zechenleitungen allein. Gegen deren einseitige Interpretation der Arbeitszeitregelung protestierten zahlreiche Belegschaften, wobei sich im Verlauf der Proteste zeigte, daß die Belegschaften in den offensichtlich einflußlosen Arbeiterausschüssen nicht mehr ihre Vertretung sahen. Vielmehr wurden neue betriebliche Ausschüsse gebildet, die ein direkteres Vorgehen der Belegschaften zu erleichtern schienen. Diese neuen Gremien wurden auch zu Sprachrohren der Belegschaften bei Lohnforderungen, die, da eine Tarifierung der Löhne zunächst nicht erfolgte, ebenfalls betrieblich artikuliert werden mußten. Die Verweigerung von Lohnzugeständnissen auf betrieblicher Ebene durch die Zechenleitungen, die unisono auf die ausstehenden Tarifverhandlungen ver- 
wiesen, bedingte vor dem Hintergrund der Kriegserfahrung den Übergang zur „direkten Aktion“, zum Streik und, da die öffentliche Autorität zusammengebrochen war, zur „direkten Aktion“ auch in Form von Zechenbesetzungen und Absetzung mißliebiger Vorgesetzter, die sich gegen die Streikenden stellten. Die Auseinandersetzungen unterschieden sich in Verlauf und Intensität dabei je nach Schachtanlage deutlich; die extreme Konfrontation in Hamborn und Oberhausen war weder bei den Südrandzechen noch im Dortmunder Raum zu finden, jedenfalls nicht im Dezember 1918. Das Versagen des Tarifsystems bzw. die lange Zeitdauer der Tarifverhandlungen und ihre in den Augen der Bergleute unzureichenden Ergebnisse brachten aber zwischen Januar und April 1919 das gesamte Revier in Aufruhr, der erst durch massiven Militäreinsatz beendet werden konnte. Die Wirkungen der Kämpfe aber waren nicht gering. Einerseits gelang es den Bergleuten, erhebliche Zugeständnisse bei Löhnen und Arbeitszeit durchzusetzen, zum anderen griffen Gewerkschaften und Reichsregierung die Streikkämpfe mit dem Ziel auf, eine politische Lösung der Spannungen im Ruhrbergbau auf doppelte Weise zu erreichen, nämlich einerseits durch die Sozialisierung der Branche, zum anderen durch die strukturelle Verbesserung der betrieblichen Kommunikation mit der offiziellen Akzeptanz der Betriebsräte als einer Weiterentwicklung der bisherigen Arbeiterausschüsse. Diese Überlegungen gingen in die richtige Richtung, vor allem eine Verbesserung der betrieblichen Kommunikation schien dringend erforderlich, jedoch versandeten beide Ansätze nicht zuletzt wegen der Halbherzigkeit der Reichsregierung, aber auch wegen des Widerstandes der Unternehmer. In beide Richtungen wurden daher gemessen an den Problemen auf den Zechen nur unzureichende Schritte unternommen. Das Tarifsystem funktionierte nach 1919 lediglich wegen des Drehens an der Kohlenpreisschraube, die Betriebsräte befanden sich recht schnell in einer ähnlichen Situation alltäglicher Blockaden wie die früheren Arbeiterausschüsse.

Kurz: Während in Leverkusen die „Revolution“ und ihre Ergebnisse (Tarif, Achtstundentag) auf der Betriebsebene in ein geregeltes, kommunikationsintensives Verfahren umgesetzt wurden, fehlte im Bergbau gerade dieses Verfahren, das die Konfliktaustragung entscheidend hätte konditionieren können. Die Verweigerungs- oder besser Passivitätshaltung der Zechenleitungen war also doppelt defizitär: sie erhöhte das Eskalationspotential und verringerte die eigenen Eingriffsmöglichkeiten, die in einem geregelten Verfahren zudem differenzierter und zielgerichteter hätten genutzt werden können. Mit der Konflikteskalation im Dezember 1918 und im Januar 1919 war freilich der Ausweg in geregelte Verfahren verschüttet, weil jetzt eine relevante Gruppe von Bergleuten sich hiervon nichts mehr versprach, sondern auf direkte Veränderungen der Eigentums- und Kontrollrechte drang. Das Verhalten der Streikenden, insbesondere die häufigen gewalttätigen Übergriffe auf das Zechenpersonal sowie die über ein verhandelbares Maß hinausreichenden Lohn- und Arbeitszeitforderungen, schienen im Nachhinein jetzt auch die Haltung vom Oktober und November 1918 zu bestätigen, so daß sich in den auch moralisch eskalierenden Konflikten eine erneute Verhärtung der Haltung der Zechenleitungen einstellte.

$\mathrm{Daß}$ indes auch die Leverkusener Regelungen nicht strukturell krisenfest waren, zeigte die Entwicklung nach 1920. Das Betriebsrätegesetz selbst legte die bis- 
herigen Arbeiterausschüsse zwar stärker an die juristische Kette, Proteste gegen seine Vorschriften blieben in Leverkusen allerdings ohne Bedeutung. Zunächst funktionierte die betriebliche Mitbestimmung daher weiter fast mustergültig. Auch wenn die jeweiligen Tarifregelungen bei bestimmten Arbeitergruppen auf Unwillen stießen, konnten die Proteste doch in den neuen Mitbestimmungsgremien vergleichsweise rasch aufgefangen werden. Erst der Steuerkonflikt vom Sommer 1920 und die Niederlage der Belegschaft gegen eine hart vorgehende Werksleitung, die regelverletzendes Verhalten mit einer flächendeckenden Aussperrung beantwortete und die Niederlage der Belegschaft zu einem formal schärferen Vorgehen in der Akkordfrage nutzte, brachten erhebliches Mißtrauen in die industriellen Beziehungen im Werk, die die geregelte Mitbestimmungsarbeit zumindest bei einem Teil der Belegschaft in Frage stellte. Dieses Mißtrauen, durch die Kompromißhaftigkeit der Mitbestimmung etwa in Fragen der Arbeitsordnung verstärkt, führte zu einem Ansatzpunkt für die Politisierung der industriellen Beziehungen, der von den Kommunisten und Syndikalisten gezielt genutzt wurde und schließlich zum Rücktritt des gemäßigten Arbeiterrates führte. Der Arbeiterrat selbst hatte bereits im Sommer 1920 die Initiative an spontan gebildete Belegschaftsvertretungen verloren und sie nur oberflächlich zurückgewinnen können. Selbst der im Januar 1921 neugewählte, radikale Arbeiterrat wurde von den radikalen Belegschaftsteilen im Arbeitszeitstreik 1921 an die Wand gespielt, die die Belegschaft in einen von Anfang an aussichtslosen Konflikt führten. Mißtrauen in die Erfolge der Mitbestimmung, Befürchtungen, die Werksleitung plane einen Generalangriff auf den Achtstundentag und die politischen Orientierungen führender Vertreter der Arbeiterschaft zusammen bedingten zunächst aber einen hohen Grad an Geschlossenheit in der Belegschaft. Da angesichts der nachlassenden Konjunktur auch die Handlungsmöglichkeiten der Werksleitung günstig waren, war auf beiden Seiten die Eskalationsbereitschaft groß. Die Bayer-Belegschaft stand in dieser Auseinandersetzung von Anfang an isoliert dar; weder gab es Gewerkschaftsunterstützung für den Streik noch größere Sympathiebekundungen anderer Belegschaften. Entscheidend für den weiteren Verlauf der Mitbestimmungspraxis wurde die Art und Weise, wie die absehbare Streikniederlage innerhalb der Belegschaft verarbeitet wurde. Da die Streikleitung nicht bereit war, den Streik als taktischen Fehler zu sehen, mußte sie notgedrungen zu Schuldzuweisungen gegenüber Werksleitung und Gewerkschaften greifen, die, je aussichtsloser der Streik wurde, um so schärfer wurden. Zu Ende des Streiks war daher nicht nur die materielle Lage der Belegschaft schlechter als zuvor, auch das Verhältnis zur Werksleitung wie zu den Gewerkschaften war dauerhaft gestört. Es dauerte zwar noch bis Ende 1922, bis mit dem Industrieverband entsprechend der kommunistischen Gewerkschaftstaktik eine alternative Gewerkschaftsorganisation gebildet wurde, die Jahre 1921/22 waren aber bereits von scharfen Machtkämpfen innerhalb der örtlichen Gewerkschaften gekennzeichnet, von den Massenaustritten aus dem Fabrikarbeiterverband nach dem Ende des Streikes noch ganz abgesehen.

Die sich nach dem Streik wiederum einstellende, einigermaßen funktionierende Mitbestimmungspraxis war daher stark moralisch und politisch belastet, zumal auch die Werksleitung den Streik politisch und moralisch auflud und der 
eigenen Belegschaft mit verstärktem Mißtrauen und einer Intensivierung der betrieblichen Verhaltenskontrollen begegnete. Damit waren auch die effektiven Spielräume der Mitbestimmung geringer geworden. Die erneute Zuspitzung der betrieblichen Auseinandersetzungen im Frühjahr und Sommer 1923 hatte aber zunächst politische Ursachen, die sich im Kampf um die Betriebsvertretung äußerten und ergriff erst im Sommer im Rahmen der Hyperinflation größere Belegschaftsteile, deren materielle Forderungen immer dringlicher wurden. Jetzt stellte sich in Leverkusen ein ähnliches Bild wie im Ruhrbergbau ein, da die Tarifverhandlungen und die betriebliche Mitbestimmung gemessen am materiellen Problemdruck im Betrieb zu langsam reagierten. Naheliegenderweise überging die Belegschaft jetzt alle regulierten Verfahren und suchte durch „direkte Aktionen" die eigenen Forderungen durchzusetzen. Hinzutrat als radikalisierendes Moment die kommunistische Generalstreiktaktik, die die Auseinandersetzungen weiter verschärfte. Wieder, wie bereits im Frühjahr 1921, nutzte die Werksleitung ihre Handlungsspielräume, diesmal freilich in offensiverer Weise, sperrte die $\mathrm{Be}$ legschaft aus und betrieb eine hochselektive Wiedereinstellungspolitik. Die betriebliche Mitbestimmung brach in diesem Kontext zusammen; große Teile der Arbeiterschaft verloren endgültig das Vertrauen in einen Konfliktregelungsmechanismus, der auch der Gegenseite offensichtlich große Handlungsspielräume gab.

Deren Handlungsspielräume waren aber nach der Zerstörung der betrieblichen Mitbestimmungspraxis größer als zuvor. Der Irrtum nicht zuletzt der kommunistischen Belegschaftsführer bestand gerade darin, die Erfolge der Mitbestimmung zu entwerten und sie an den gewünschten strukturellen Machtveränderungen zu messen. Die Beschränkungen der Handlungsmöglichkeiten der Unternehmensleitung durch die betriebliche Mitbestimmung wurden auf diese Weise nicht wahrgenommen. Zwei Punkte waren dabei von entscheidender, aber unterschätzter Bedeutung. Einerseits brachte das „Patt“ in den industriellen Beziehungen zwischen 1918 und 1920 eine Situation, in der die bisherige Leistungslohn- und Akkordpolitik nicht mehr durchsetzbar war. Dies zwang die Werksleitung, neue Leistungslohn- und Arbeitszeitkonzepte zu entwickeln, für die sie aber kaum definitive Vorgaben besaß. Zugleich scheute sie davor zurück, nicht erprobte Konzepte einfach durchzusetzen, so sie es denn überhaupt gekonnt hätte. In dieser ungeklärten Situation zeigte sich die Werksleitung durchaus kompromißbereit, doch wurde diese Kompromißbereitschaft letztlich nicht auf die Probe gestellt. Der Steuerkonflikt und der Februarstreik zerstörten hingegen nicht nur die Kompromißbereitschaft der Werksleitung, sie verbesserte auch deren einseitige Durchsetzungsmöglichkeiten, so daß zahlreichen Belegschaftsangehörigen angesichts nicht mehr existenter Mitbestimmungsmöglichkeiten lediglich der Ausweg in die individuelle oder kollektive Unterlaufung neuer Vorschriften blieb, was im Gegenzug wiederum durch eine Verschärfung der Akkordpolitik beantwortet wurde. Wirkten sich diese Faktoren während der Inflation noch nicht voll aus, so sollte ihre Bedeutung nach 1924 erheblich wachsen.

Im Bergbau waren die Jahre zwischen 1920 und 1923 nicht einmal ansatzweise von einer funktionierenden Mitbestimmungspraxis gekennzeichnet. Anders als in Leverkusen, wo es sehr rasch um die Ausfüllung der Bestimmungen des Betriebs- 
rätegesetzes ging, verharrte die Auseinandersetzung auf den Schachtanlagen an der Ruhr bei der Definition von Rechten und Verhaltensmöglichkeiten der Betriebsräte, hinter die materielle Mitbestimmungsfragen weitgehend zurücktraten. Spielten sie doch eine Rolle, so war ihre Diskussion jeweils von den Versuchen insbesondere der Zechenleitungen gekennzeichnet, materiellen Entscheidungen aus dem Weg zu gehen. An der Ruhr stritt man sich nicht im Rahmen des Betriebsrätegesetzes, sondern über seine betriebsspezifische Umsetzung. Dabei spielten die Zechenleitungen keine konstruktive Rolle. Angesichts des Fehlens eigener sozialpolitischer Instanzen delegierten sie die einschlägigen Fragen an den Zechenverband, der zu einer streng legalistischen, faktisch restriktiven Haltung aufforderte, die von den Zechenleitungen strikt exekutiert wurde. Als bergbauliches Spezifikum erwies sich in diesem Zusammenhang die Möglichkeit der Zechenleitungen, durch Verweigerung von Befahrungsrechten, die Kommunikation zwischen Betriebsräten und Belegschaften wirksam zu behindern. Um die Befahrungsrechte einerseits, die konkreten Handlungsmöglichkeiten der Betriebsräte (Organisationskontrolle, Rechte bei Strafen und Überarbeit, Mitwirkung bei Unfällen und in der betrieblichen Sozialpolitik) andererseits tobte daher ein andauernder Streit, der auch durch tarifvertragliche Betriebsräteregeln vom Sommer 1920 nicht beendet wurde.

$\mathrm{Zu}$ eigentlicher Mitbestimmung kam es daher nicht, weil es keine allgemein akzeptierten Mitbestimmungsregeln gab. Der gegenseitige Vorwurf des Regelverstoßes ersetzte die inhaltliche Diskussion. Kam es trotz dieser Blockaden zu materiellen Auseinandersetzungen, suchten die Zechenverwaltungen durch Kleinlichkeit und Verzögerungen Entscheidungen aus dem Wege zu gehen, wenn sie die Forderungen und Wünsche der Belegschaften nicht ohnehin ablehnten. Da zugleich das Tarifsystem nur mangelhaft und schleppend funktionierte, erhielt sich angesichts guter Konjunktur und verbreiteten Energiemangels in der Bergarbeiterschaft die Tendenz, Konflikte durch „direkte Aktionen“ zu lösen, die auf Seiten der Zechenverwaltungen stets den Ruf nach staatlicher Repression der Bergarbeiterbewegung nach sich zogen. Die seit 1920 vermehrt gewählten unionistischen und syndikalistischen Betriebsratsmitglieder legten schließlich ohnehin keinen Wert auf geregelte Mitbestimmungsverfahren, sondern begriffen sich explizit politisch. Da aber auch die gemäßigten Betriebsräte keinerlei praktische Erfolge vorzuweisen hatten, kam im Bergbau endlich die betriebliche Mitbestimmung generell in Verruf.

Kohlenknappheit und Vollbeschäftigung bei geringen Leistungen zwangen andererseits die Zechenverwaltungen zu Reaktionen. Da man aus den freilich selbstprovozierten Revolutionserfahrungen heraus auf geregelte betriebliche Konfliktverfahren keinen Wert legte, besaß man in der Frage der Leistungssteigerung und der Disziplinierung der Belegschaften keinerlei Handhabe, abgesehen vom Ruf nach staatlicher Repression. Weil der Zechenverband zudem zu materiellen Zugeständnissen nur nach langen Verhandlungen und Erhöhungen der Kohlenpreise bereit war, beruhte das gesamte fragile Gleichgewicht des Bergbaus nach 1920 auf der Inflation und der Bereitschaft der Tarifpartner, die Inflation zur Überbrückung ihrer Konflikte zu nutzen. Bereits jetzt zeichnete sich die Konstellation ab, daß der Bergbau eine unbestreitbare notwendige Erhöhung der Förder- 
leistungen nur gegen die Bergarbeiter, nicht in Kooperation mit ihnen durchsetzen wollte.

Den Hintergrund dieser Vorstellungen einer autonom zu gehenden Strategie der Produktivitätssteigerung bildeten bestimmte Überzeugungen, die einerseits mit den technischen Bedingungen des Bergbaus, andererseits mit der Führungstradition und den Revolutionserfahrungen der Zechen zusammenhingen. War man im Gegensatz zu den Leverkusener Farbwerken über die anzuwendenden Leistungslohnsysteme zu keinem Zeitpunkt im Zweifel - das Gedinge als Stücklohnform erwies sich trotz der Bergarbeiterwiderstände gegen die bisherigen Gedingeformen zu keinem Zeitpunkt als grundsätzlich überholt -, so hatte andererseits die nicht zuletzt über das Gedinge sichergestellte autoritäre Integration der Arbeiterschaft in den Bergbaubetrieb sich als die angemessene Arbeitsorganisation der Vorkriegszeit erwiesen, die nicht durch arbeits- und sozialpolitische Überlegungen differenziert worden war. Das Fehlen derartiger Ausdifferenzierungen auch in der eigenen Selbstbeobachtung und die Überzeugung von der technischen Angemessenheit der Arbeitsorganisation im Bergbau bedingte $a b$ 1916, verstärkt ab 1918/9 auch eine Wahrnehmung der Bergarbeiterbewegung, die auf grobe Verzerrungen des Bildes hinauslaufen mußte. Spätestens mit dem starken Rückgang der Produktivität trat zudem die betriebswirtschaftliche Lage der Zechen als weiterer Punkt hinzu, der Leistungssteigerungen um jeden Preis zu verlangen schien, spätestens dann, wenn der inflationäre Schleier wegfallen würde und die Zechen wieder unter normalen Marktbedingungen arbeiten mußten.

Das feste Bewußtsein, einen Weg aus der Krise zu wissen, der durch das kollektive Arbeitsrecht und die Betriebsrätegesetzgebung nur in Frage gestellt wurde, ließ den Zechenleitungen daher ein Arrangement mit den neuen arbeitsrechtlichen Strukturen als gefährlich und kostenträchtig erscheinen. Die Erfahrung einer in sich zerstrittenen und keineswegs einheitlichen Bergarbeiterschaft schien zudem die Auffassung zu bestätigen, die widerständigen Teile der Belegschaften und ihre Wortführer repräsentierten keineswegs die Bergarbeiterschaft, sondern könnten unter günstigen Bedingungen „ausgesiebt“" werden. Waren wegen der guten Konjunktur und der Vollbeschäftigung die Handlungsmöglichkeiten der Zechen zunächst stark beschränkt, so änderte sich diese Situation in der Hyperinflation. Die zusammenbrechende Nachfrage ermöglichte eine Disziplinierung der Belegschaften über das Arbeitsmarktrisiko und schuf damit Spielräume, die eigenen Vorstellungen gegen die Bergarbeiterschaft durchsetzen zu können. Zudem konnte man endlich mit der so lange herbeigesehnten „Säuberung“ der Belegschaften beginnen.

Die Jahre zwischen 1924 und 1929 waren vor dem Hintergrund der Strukturprobleme des Weltmarktes sowohl im Bergbau wie in der Chemischen Industrie von grundlegenden Auseinandersetzungen um die Kosten des Arbeitsprozesses gekennzeichnet. In beiden Branchen versuchten die Unternehmensleitungen, die Arbeitsproduktivität entscheidend zu erhöhen und die Produktionskosten zu senken, um auf den hart umkämpften Weltmärkten wieder Fuß fassen zu können. Im Gegenzug trachteten Gewerkschaften und Belegschaften danach, die materiellen Lebensbedingungen der Arbeiterschaft nach deren Tiefstand während und nach der Währungsanpassung zu verbessern. Arbeitszeiten und Leistungslohn- 
systeme wurden daher zu den Brennpunkten betrieblicher sozialer Auseinandersetzungen.

Die Konfliktverläufe unterschieden sich in beiden Branchen erheblich. Einerseits befanden sich die Leverkusener Farbwerke in einer ökonomisch günstigeren Situation als der strukturkrisengeschüttelte Bergbau, woraus sich ein Teil der Verbissenheit der Politik der Zechenleitungen erklärt; andererseits waren in der Chemischen Industrie die Vorstellungen über die Leistungslohnsysteme und deren Anwendbarkeit im Betrieb nicht derart festgelegt wie im Bergbau; drittens schließlich variierten Ausmaß und Art der Rationalisierung im technischen Bereich zum Teil deutlich von einander. Viertens schließlich hatte sich in der Chemischen Industrie nach 1918 das Tarifsystem schnell eingespielt und bewährt, während es im Bereich des Bergbaus heftig umstritten blieb und lediglich wegen der Nutzung der Preisspirale nicht zusammenbrach. Die betriebliche Mitbestimmung, um einen letzten Punkt zu nennen, war zwar auch in Leverkusen 1921 bis 1923 sukzessive in Frage gestellt worden und schließlich zusammengebrochen, doch resultierte dieser Zusammenbruch weniger aus einer grundsätzlich obstruktiven Haltung der Werksleitung, wie sie für den Ruhrbergbau typisch war, als vielmehr aus der Zuspitzung sozialer Konflikte im Betrieb, die die betriebliche Mitbestimmung an den Rand drückten und ihre Bedeutung stark verringerten.

Der Mitbestimmungsalltag in Leverkusen litt noch bis 1925/6 unter den Nachwirkungen der Kämpfe während der Hyperinflation, da die Belegschaft nicht zuletzt auch aus der Erfahrung der Anpassungskrise, in der im übrigen der noch gemäßigte Betriebsrat eng mit der Werksleitung etwa bei der Verlängerung der Arbeitszeit zusammengearbeitet hatte, linksradikal votierte. Dem Betriebs- und Arbeiterrat, den mehrheitlich der kommunistische Industrieverband bildete, versagte die Werksleitung jede produktive Kooperation, zumal ihre Kooperationsbereitschaft mit den Belegschaftsvertretungen auch generell auf Kritik namentlich im mittleren Management traf, das die Kompromißbereitschaft gegenüber der $\mathrm{Be}-$ triebsvertretung für Reibungsverluste in der betrieblichen Arbeitsorganisation verantwortlich machte. Zwischen 1924 und 1926 kam es daher lediglich zu einer informellen Form betrieblicher Mitbestimmung zwischen der Sozialabteilung und den gemäßigten Belegschaftsvertretern, deren Ausmaß sich aber in keiner Weise mehr mit der intensiven Zusammenarbeit der Jahre 1918 bis 1920 vergleichen ließ. Erst nach Wahlerfolgen der Tarifgewerkschaften verbesserte sich zwischen 1926 und 1929 erneut die betriebliche Kooperation, deren Reichweite jedoch begrenzt blieb, da die Werksleitung nach 1924 mit einer autonom geplanten und durchgesetzten Form der Akkordierung der Arbeitsprozesse durchaus erfolgreich gewesen war. Spielräume für grundsätzliche Debatten in der Akkordfrage existierten nach 1926 kaum noch, die Betriebsvertretung konnte lediglich jeweils im Nachhinein gewisse Korrekturen in der betrieblichen Akkordpolitik durchsetzen.

Die industriellen Beziehungen in Leverkusen nach 1924 waren gleichwohl durch den Akkordkonflikt bestimmt. Der Versuch, die Arbeitsproduktivität zu steigern, stieß auf den Widerstand der Belegschaft, die ihr Arbeitsverhalten jeweils an "gerecht" erscheinenden Akkordmehrverdiensten orientierte, in den Augen 
der Werksleitung daher Akkordbremsen betrieb. Diese Konflikte wurden nun aber kaum mehr zum Gegenstand betrieblicher Aushandlungsprozesse, vielmehr suchte die Werksleitung zunächst durch den Stückakkord, danach durch präzise Vorgaben im Zeitakkord das Akkordbremsen zu bekämpfen. Dies bedingte zugleich eine Aufwertung der Arbeitsvorbereitung durch Zeitprüfer und -kalkulatoren, andererseits eine Abwertung der bisher in der Akkordkalkulation entscheidenden Rolle der Meister. Mit diesen Veränderungen waren zwar ebenfalls Reibungsverluste verbunden, die der Betriebsrat thematisierte, doch waren in den Augen der Werksleitung die Reibungsverluste geringer als die Vorteile der neuen Akkordformen. Ein Zwang zur Revision der Akkordpolitik bestand in den Augen der Werksleitung daher zu keinem Zeitpunkt; auch ein breiter Widerstand der Belegschaft war angesichts des nach 1924 deutlich gewachsenen Arbeitsmarktrisikos unwahrscheinlich, so daß dem Betriebsrat die Ansatzpunkte für eine effektive Einflußnahme auf die Akkordpolitik des Werkes fehlten. Durch das vergleichsweise reibungslose Funktionieren des Tarifsystems spielten Fragen der Arbeitszeit nach 1924 keine wesentliche Rolle mehr. Die Verlängerung der Regelarbeitszeit um eine Stunde Anfang 1924 war zudem gemeinsam mit dem 1922 gewählten, gemäßigten Arbeiterrat durchgeführt worden. Die linksradikale Betriebsvertretung versuchte 1924/5 vergeblich, die Belegschaft gegen die Arbeitszeitverlängerung zu mobilisieren. Widerstände dürften auch hier im wesentlichen durch das Arbeitsmarktrisiko diszipliniert worden sein. Seit 1926 wurde überdies die traditionell ausgeprägte betriebliche Sozialpolitik wieder verstärkt zur Integration der Arbeiterschaft in den Betrieb genutzt. Dem Betriebsrat blieb nach 1926 daher abgesehen von der Feinanpassung der Tariflöhne an die Arbeitsverhältnisse in Leverkusen kaum ein Gebiet, auf dem er erfolgreich hätte agieren können.

Im Bergbau stellte sich zu Beginn der Weltwirtschaftskrise eine ähnliche Situation ein, jedoch mit einer erheblich anderen Vorgeschichte. Die Anpassungskrise 1923/24 nutzten die Zechenleitungen gezielt zur „Säuberung“ der Belegschaften und zur weiteren Paralysierung der Betriebsratstätigkeit. Die Wahlergebnisse zu den Betriebsräten, die bis 1925 relevante unionistische und syndikalistische Stimmenanteile brachten, waren allerdings Ausdruck dafür, daß diese Paralysierung und Säuberung zunächst nicht durchgreifend erfolgreich war. Der Kampf gegen die Betriebsräte ging daher bis 1925 weiter; aber auch nach dem Ende der "Union“ versuchten die Zechengesellschaften weiterhin erfolgreich, eine geregelte Mitbestimmungspraxis zu verhindern. Anders als in der Chemie stellte sich im Bergbau auch nach 1924 keine Situation dem Grundsatz nach akzeptierter Mitbestimmungsverfahren ein. Die Auseinandersetzung um die Befahrungsrechte und der Streit um die Ausstattung von Betriebsratsbüros, Sprechstunden, Aushänge, überhaupt um die gesamte Kommunikation zwischen Betriebsvertretung und Belegschaft dauerte bis 1929 an. Eine gemeinsame Diskussion der betrieblichen Probleme unterblieb, da die Zechenleitungen den Betriebsvertretungen die Artikulationsmöglichkeiten hierfür nahmen. Die Folgen der Leistungssteigerungs- und Rationalisierungspolitik wurden daher in den Betrieben nicht geregelt verarbeitet. Zunächst schien dieses Vorgehen der Zechenleitungen durchaus erfolgreich zu sein, da das Arbeitsmarktrisiko die Widerstandsbereitschaft der Bergleute verringerte und die zahlreichen Feierschichten die Bereitschaft zur Mehrarbeit erhöh- 
ten. Die individuelle Arbeitsproduktivität stieg zugleich wegen der rationalisierungsbedingten Veränderungen im Bergbau an. Parallel zur Leistungsintensivierung nahm allerdings das unregulierte Konfliktverhalten der Bergleute zu; einerseits machten sich Abwanderungstendenzen qualifizierter Arbeitskräfte bemerkbar, andererseits nutzten mehr und mehr Bergleute die gewachsenen Spielräume der Kranken- und Arbeitslosenversicherung, um die negativen Folgen der Arbeitsintensivierung und der zahlreichen Feierschichten auszugleichen. Nach Berechnungen der Zechenleitungen neutralisierten sich Krankenzeiten und Mehrarbeit sogar gegenseitig. Das Klima im Bergbau blieb daher von sozialen Spannungen in einem Maße beherrscht, daß jederzeit erneut mit dem Ausbruch von Unruhen gerechnet werden mußte, auch wenn dies wegen der Arbeitsmarktlage unwahrscheinlich war. Dabei waren es in den Augen der Zechenleitungen vor allem die Betriebsräte, von denen eine Gefahr drohte.

Die Folgen des englischen Bergarbeiterstreikes deckten zahlreiche ungelöste Probleme des Bergbaus auf. Die Abwanderung qualifizierter Bergarbeiter und die Vernachlässigung der Nachwuchsförderung brachten jetzt das Problem des $\mathrm{Ar}$ beitskräftemangels. Die gute Konjunktur konnte nur mit Mehrarbeit ausgenutzt werden, zu der die Mehrzahl der Bergleute zumindest zeitweilig auch bereit war. Bereits im Herbst 1926 aber ließ wegen offensichtlicher Erschöpfung der Bergleute die Bereitschaft zur Mehrarbeit nach; die Entwicklung der Arbeitsproduktivität, die von 1924 auf 1926 stark angestiegen war, stagnierte; Kranken- und Unfallziffern schossen in die Höhe. Anzeichen für eine wieder zunehmende Konfliktbereitschaft der Bergleute häuften sich. Die rigorose Gedingepolitik einzelner Zechen wurde ihnen nun zum Verhängnis, da die Fluktuation der Bergleute stark zunahm, abgekehrte Bergleute aber vom Arbeitsmarkt nicht mehr ersetzt werden konnten. Spätestens jetzt wurde deutlich, daß eine Rationalisierungs- und Leistungssteigerungspolitik, die nicht auf einer realistischen Wahrnehmung der Lebensbedingungen der Bergleute beruhte, dauerhaft nicht erfolgreich sein würde.

Anfang 1927 standen die Zechenleitungen daher vor einem Dilemma. Blieb es bei der guten Konjunktur, so würden die Bergleute bei den anstehenden Lohnund Manteltarifverhandlungen u.U. weitreichende Zugeständnisse in der Arbeitszeit- und Lohnfrage durchsetzen können, die man betriebswirtschaftlich für unverantwortbar hielt. Diese Situation war durch die Preispolitik des RWKS in gewisser Weise mit herbeigeführt worden, da das Syndikat den Ausfall der englischen Kohle weniger zu Preiserhöhungen als zum Abschluß langfristiger Lieferverträge bei vergleichsweise moderaten Preisen genutzt hatte, die nur bei einer hohen Auslastung der Zechen gewinnträchtig waren. Hohe Löhne und kürzere Arbeitszeiten stellten einerseits die Auslastung der Gruben in Frage - im Winter 1926/27 war bereits eine volle Ausnutzung der Anlagen wegen des Arbeitskräftemangels nicht möglich; Arbeitszeitverkürzungen würden den Arbeitskräftemangel und damit den Förderausfall weiter erhöhen, so lange nicht genügend qualifizierte Hauer zu bekommen waren -, andererseits drückten sie auf die angesichts des niedrigen Preisniveaus ohnehin nicht sehr großen Gewinnmargen. Unterblieben Zugeständnisse, so war mit Abwanderungen, Unruhen und weiter zunehmender Fluktuation zu rechnen, die Bereitschaft zur Mehrarbeit würde weiter zurückgehen, evt. war sogar mit Streiks zu rechnen. 
Spätestens in dieser dilemmatischen Situation hätten die Zechenleitungen erkennen müssen, daß an einem kooperativen Ausgleich mit der Bergarbeiterschaft kein Weg vorbeiführte. Da diese Kooperation indes materielle Zugeständnisse impliziert hätte, sträubten sich die Zechenleitungen nachhaltig gegen einen Interessenausgleich und versuchten, durch Einflußnahme auf die Politik ihren Standpunkt durchzusetzen. Dies gelang ihnen allerdings nur teilweise, die Schiedssprüche vom März und April 1927 schrieben die bisherigen Arbeitszeiten fest und brachten eine moderate Erhöhung insbesondere der Hauerlöhne. Zu diesem relativen Erfolg der Zechenleitungen trug vor allem bei, daß sich im Frühjahr 1927 erneut Strukturkrisenzeichen insbesondere bei den „reinen“ Zechen bemerkbar machten, die Zahl der Feierschichten zunahm und das Arbeitsmarktrisiko der Bergleute wuchs. Vorhandene Bergarbeiterwiderstände gegen die in ihren Augen unzureichenden Tarifabschlüsse unterblieben daher weitgehend.

Statt dessen schoben sich die seit längerem vorhandenen und erkennbaren Spaltungen der Bergarbeiterschaft in den Vordergrund. Die Abwanderung aus dem Bergbau nahm immer größere Züge an. Die Arbeitslosigkeit wurde auf diese Weise gering gehalten, die Lage der verbleibenden Bergleute blieb materiell erträglich, offene Widerstände gegen die Politik der Zechenleitungen beschränkten sich auf die politisierten Bergleute. Die Betriebsräte, zerrissen zwischen Belegschaftsforderungen, Gewerkschaftsprogrammatik und Feindseligkeit der Zechenleitungen hatten nur noch wenig effektive Handlungsmöglichkeiten. Insbesondere versagten ihnen die Zechenleitungen jeden Erfolg bei der Bewältigung der Rationalisierungsfolgen, so insbesondere bei der Pausenregelung, der Gedingestellung und der Mindermaßkontrolle.

Die forcierte Rationalisierung nach dem Ende des Bergarbeiterstreikes brachte den Betriebsräten allerdings auch neue Aufgaben. Massenentlassungen und Stilllegungen waren nach den Vorschriften der Demobilmachungsverordnung „mitbestimmungspflichtig", zumindest wenn dabei mehr als 50 Bergleute ihren Arbeitsplatz verloren. Auch wenn sie es versuchten, konnten die Zechen nicht jede Verhandlung verhindern. Die Tätigkeit der Betriebsräte, die bei der Planung und Durchführung von Rationalisierungsmaßnahmen keine Stimme hatten, beschränkte sich freilich auf die sozial verträgliche Auswahl der zu Entlassenden, was bei den kontinuierlichen Massenentlassungen schließlich kaum noch möglich war. Vorzeigbare Erfolge konnten daher auch in diesem Bereich nicht erzielt werden.

Als Antwort auf die sich 1927 wiederum verschlechternde Absatzlage intensivierten die Zechen, vor allen die Abteilung Bergbau der VSt. ihre Rationalisierungsbemühungen weiter, die überdies den Vorteil zu haben schienen, aus dem Dilemma der Arbeitskräfteknappheit des Frühjahres 1927 herauszuführen. Erste Erfolge stellten sich 1928/29 ein; die betriebswirtschaftlichen Daten der Abteilung Bergbau der VSt. verbesserten sich deutlich; die Gewinnsituation entwickelte sich so günstig, daß man sogar aus der bisherigen Politik des RWKS der Exportförderung ausscherte und sich für eine Kürzung des umlageintensiven Exportes aussprach. Offensichtlich glaubte die Abteilung Bergbau, bei einer zumindest teilweise erfolgenden Demontage des RWKS als Unternehmen von der dann voll ausbrechenden Strukturkrise nur profitieren zu können. Jedenfalls war man zu einer 
Subventionierung der „Grenzzechen“, die an der Rentabilitätsgrenze arbeiteten, über das Umlageverfahren des RWKS nicht mehr im bisherigen Ausmaß bereit. In gleicher Weise hatte die Rationalisierung auch größere Handlungsspielräume gegenüber den Belegschaften geschaffen, von deren Arbeitswilligkeit man bei der verbesserten Fördertechnik nicht mehr im gleichen Ausmaß wie früher abhängig war. Zugeständnisse gegenüber Gewerkschaften und Betriebsräten schienen im Gegenteil die Rationalisierungserfolge zu gefährden. Den Ruhreisenstreit im November 1928 nutzte daher auch die im Zechenverband ohnehin tonangebende Abteilung Bergbau der VSt. zu einem Grundsatzangriff auf das Tarifsystem.

Daß sich die Stimmung in den Belegschaften 1928 und 1929 weiter verschlechterte und auch das Verhältnis zwischen Vorgesetzten und Kameradschaften schlecht war, sah man gleichwohl mit Befürchtungen, eine erneute Eskalation der sozialen Auseinandersetzungen sei nicht auszuschließen. Kommunistische Wahlerfolge bei den Betriebsratswahlen 1929 bestätigten diese Befürchtungen. Da man indes mit der bisherigen Rationalisierungspolitik durchaus erfolgreich war, suchte man nach einem Konzept zur sozialen Integration der Belegschaften, ohne an der Betriebsführungsstrategie Änderungen vornehmen zu müssen. Die Propagierung der Werksgemeinschaftsidee bekam daher die Funktion, eine symbolische Integration der Belegschaften zu erreichen, die man materiell nicht glaubte bewerkstelligen zu können. Zu Beginn der Weltwirtschaftskrise war damit im Bergbau nicht nur die betriebliche Mitbestimmung praktisch gescheitert; die führenden Protagonisten des Bergbaus plädierten zudem immer offener für deren restlose Beseitigung und boten mit der Werksgemeinschaft eine scheinbar funktionale Alternative hierzu an. Die bisher nur negativ verstandene Bekämpfung der Betriebsräte wurde auf diese Weise um ein offensives Konzept erweitert, das freilich in einer parlamentarischen Demokratie kaum Realisierungschancen besaß.

Von betrieblicher Mitbestimmung konnte in der Weltwirtschaftskrise nicht mehr gesprochen werden. Auch wenn das Bild in der chemischen Industrie und im Bergbau ähnlich war, so waren doch die zugrundeliegenden Verhältnisse unterschiedlich. Insbesondere betrieb in Leverkusen die Werksleitung nicht die grundsätzliche Beseitigung der Betriebsräte, deren Gewicht ohnehin deutlich gesunken war. Die Leverkusener Werksleitung verfolgte in der Weltwirtschaftskrise ihr bisheriges Ziel, die Leistungsfähigkeit des Werkes durch Erhöhung der Arbeitsproduktivität zu steigern, weiter fort. Da man mit dieser Politik bereits in den Jahren zuvor sehr erfolgreich gewesen war und die Belegschaftszahl niedrig hatte halten können, versuchte die Werksleitung während der Weltwirtschaftskrise weniger über Entlassungen als mit Arbeitszeitverkürzungen das Arbeitsvolumen an die sinkende Produktion anzupassen. Gleichwohl erfolgten in Schüben größere Entlassungsaktionen, so daß die verbleibende Belegschaft trotz relativer Arbeitsplatzsicherheit den Verlust des Arbeitsplatzes nicht ausschließen konnte. Entsprach man mit Arbeitszeitverkürzungen statt Entlassungen ohnehin den gewerkschaftlichen und den Vorstellungen der Betriebsräte, so nahm andererseits auch die drastisch sinkende Konfliktbereitschaft der Belegschaft dem Betriebsrat faktisch jede Handlungsmöglichkeit.

Für die Belegschaft wurde die Weltwirtschaftskrise daher zu einer Phase stark zurückgehender Einkommen bei gleichzeitiger Unmöglichkeit, die eigenen drän- 
genden Interessen noch formulieren und durchsetzen zu können. Diese Kluft wurde zumindest teilweise durch die Wahl radikaler Gruppen überbrückt, die die Mißstimmung der Belegschaften verbal bedienten, angesichts der nicht vorhandenen Handlungsbereitschaft der Belegschaften sich aber selbst ebenfalls auf verbalradikale Aktionen beschränken mußten. Diese Aktionen trugen indes zur Delegitimierung der letzten Reste der betrieblichen Mitbestimmung nicht unwesentlich bei, an deren Beseitigung in Leverkusen nach 1933 vor allem die völlige Reibungslosigkeit auffällig war, mit welcher der Übergang von den gewählten zu den ernannten Nazi-Betriebsräten vollzogen wurde. Die Demontage der Mitbestimmung bei der Belegschaft war schon früher erfolgt; 1933 dürfte deren Interesse stärker durch die sich bessernde Konjunktur, die längeren Arbeitszeiten und damit die wieder wachsenden Einkommen bestimmt worden sein.

Die lange Zeit enttäuschten Erwartungen der Belegschaft, durch Tarifsystem und betriebliche Mitbestimmung materielle Vorteile erreichen zu können, wurden vom Konjunkturaufschwung erfüllt. Materielle Vorteile waren nun auch ohne Betriebsräte und Gewerkschaften spürbar, auch wenn sie die Rechtlosigkeit der Belegschaften kaum kaschiert haben dürften. Nach vier Jahren Krise aber wogen Arbeitsplatzsicherheit und ausreichende Einkommen mehr als die Hoffnung auf Rechte, die letztlich lediglich darin zu bestehen schienen, über die Löhne und Arbeitsbedingungen mitreden zu dürfen, ohne daß dieses Mitreden greifbare Erfolge gebracht hätte. Es war wegen der Konjunkturlage ohnehin unwahrscheinlich, daß die Belegschaft einen offenen Konflikt um das Betriebsrätegesetz riskiert hätte. Angesichts seiner scheinbar nur geringen Erfolge verblaßte es zudem hinter der Wirkung des Konjunkturaufschwunges. Werksleitung und Belegschaft in Leverkusen jedenfalls arrangierten sich 1933/34 ohne größere Probleme mit der neuen Situation, zu deren Heraufkunft man wenig beigetragen hatte: Bei der Gruppe Niederrhein der I.G. Farben hätte man auch mit dem Betriebsrätegesetz weiterleben können.

Im Bergbau waren die Verhältnisse komplizierter, im Prinzip aber denen in Leverkusen nicht unähnlich. Der Einbruch der Kohlenförderung in der Weltwirtschaftskrise führte zu einem scharfen Personalabbau. Die verbleibenden Bergarbeiter litten unter zahlreichen Feierschichten, sinkenden Einkommen und hohem Arbeitsmarktrisiko. Verheerend wirkte dabei, daß die Massenentlassungen zu einem dauerhaften Phänomen wurden, die Belegschaftsverringerung also sukzessive und nicht zu einem einmaligen Zeitpunkt erfolgte. Die Bergarbeiter, zu einem großen Teil ohnehin bereits vor 1929 über ihre Zukunftsaussichten zutiefst verunsichert und politisch resigniert, vom Tarifsystem und der Kohlengemeinwirtschaft, die die Folgen der Rationalisierung kaum hatten mildern können, enttäuscht, verloren in der Krise jede Möglichkeit der Konfliktaustragung. Vor der Krise hatte es zumindest die Chance zur Abwanderung, zur Fluktuation oder zum "wilden" Feiern gegeben. Nach 1929 traute man sich nicht einmal mehr, kleinere Unfälle anzugeben oder „zu“ häufig zum Arzt zu gehen. Beschwerden gegenüber Vorgesetzten unterblieben völlig, obwohl oder gerade weil deren Auftreten in der Weltwirtschaftskrise wieder autoritärer wurde.

Denn die Leitung der Abteilung Bergbau der VSt. nutzte die Weltwirtschaftskrise nicht nur zur letztlich erfolgreichen Bekämpfung des Tarifsystems, sondern 
trieb auch die Rationalisierung und Kosteneinsparung weiter voran. Allein betriebswirtschaftlich gesehen, stellte die Weltwirtschaftskrise sogar die Sanierungsphase des Konzerns dar, der 1933/34 allen wichtigen Parametern nach die Kostenkrise der zwanziger Jahre überwunden hatte. Die Angst, im Aufschwung erneut zu Kostenerhöhungen über Tarife und betriebliche Mitbestimmung gezwungen zu sein, verhärtete die scharfe Frontstellung gegen das bisherige Arbeitsrecht. In der Weltwirtschaftskrise konnte das autoritäre Werksgemeinschaftskonzept daher eine hohe Wirkung entfalten und stand letztlich bei der Abschaffung des Betriebsrätegesetzes Pate. Wie sehr man dabei im Bergbau auf die Kostenträchtigkeit der neuen Arbeitsgesetzgebung achtete, zeigte sich noch einmal bei der illegalen Verdrängung der gewählten Betriebsräte. Der Zechenverband verlangte eine nachträgliche Legalisierung aller Maßnahmen nach dem Januar 1933, um sich möglichen Regreßansprüchen zu Unrecht abgesetzter und entlassener Betriebsräte entziehen zu können. Versuche der neuen Nazi-Betriebsräte, Interessenpolitik für die Bergarbeiter zu betreiben, begegnete man mit der bekannten Schärfe.

Da die anziehende Konjunktur im Bergbau zunächst die Arbeitslosigkeit nicht beseitigte, funktionierte das neue Arbeitsrecht scheinbar. Die Ruhe unter den Bergleuten wurde allerdings weniger vom AOG als vom Arbeitsmarktrisiko garantiert. Nach 1936 kehrte in den Bergbau daher die alte Konstellation zurück, daß die Bergleute bei fehlenden Konfliktregulierungsverfahren den Weg in die individuelle Konfliktaustragung suchten. $\mathrm{Zu}$ deren Disziplinierung mußte der politische Zugriff auf das Arbeitsverhältnis dauernd erweitert werden, bis schließlich die Arbeitsvertragsfreiheit völlig beseitigt war. Der wirtschaftlich begründete Verzicht auf eigene Integrations- und Anpassungsleistungen in der Sozial- und Arbeitspolitik hatte bereits in der Weimarer Zeit die Handlungsfähigkeit der Zechenleitungen deutlich gesenkt; nach 1933 war man schließlich völlig auf politische Hilfe angewiesen, um das Verhältnis zur eigenen Arbeiterschaft noch steuern zu können.

\section{Die „Determinanten“ der Konfliktentwicklung}

Versucht man aus der Geschichte der industriellen Beziehungen in Leverkusen und im Ruhrbergbau jene Faktoren herauszuarbeiten, die die Konfliktentwicklung und damit Art und Ausmaß der betrieblichen Entwicklung entscheidend beeinflußten, so fallen insgesamt mehrere Punkte ins Auge. Die Isolierung dieser Punkte soll ihnen nicht unbedingt ursächliche Bedeutung für den Konfliktverlauf geben, sondern sie als einflußreich hervorheben. Ihre konkrete Bedeutung war im Konfliktverlauf wiederum Wandlungen unterworfen, wie überhaupt eine Ursache-Wirkungsanalyse stets in der Gefahr ist, Kausalitäten zu konstruieren anstatt nachzuweisen. Im folgenden soll es daher vor allem darum gehen, die Entwicklung der industriellen Beziehungen im Betrieb jeweils unter spezifischen Fragestellungen $\mathrm{zu}$ interpretieren.

1. Entscheidende Bedeutung für die Entwicklung der industriellen Beziehungen im Betrieb kam ohne Frage der wirtschaftlichen Entwicklung zu. Die wirtschaftliche Entwicklung schuf die Rahmenbedingungen, auf die die Unternehmen 
durch Anpassung oder Verweigerung von Anpassung zu reagieren hatten, sie bedingte Art und Ausmaß der jeweiligen Zieldefinitionen der beteiligten Akteure und sie entschied letztlich über die jeweils verfügbaren Machtressourcen, diese Zielvorstellungen im betrieblichen Rahmen durchzusetzen.

Im Untersuchungszeitraum lassen sich dabei zwei Phasen von einander unterscheiden. Die erste Phase umfaßte den Zeitraum von 1916 bis 1923 und war trotz zeitweiliger Schwankungen von Hochkonjunktur, Produkt- und Arbeitskräftemangel gekennzeichnet. Der inflationsbedingte Geldschleier behinderte in den Betrieben überdies eine exakte Betriebswirtschaft und Leistungskontrolle. In diesen sieben Jahren waren damit die Ausgangspositionen der Belegschaften zur Durchsetzung von Arbeitszeitverkürzungen und Lohnerhöhungen günstig, andererseits standen den Arbeitgebern erhebliche Widerstände bei der Durchsetzung leistungssteigernder Maßnahmen und der damit verbundenen Kostensenkungsaktionen entgegen. $\mathrm{Da}$ in diesem Zeitraum allerdings der Druck von den Märkten inflationsbedingt gering war, versuchten die Arbeitgeber vorrangig, sich mit den Belegschaften zu arrangieren, um die günstige Konjunktur nutzen zu können. Zeitweilige Konjunktureinbrüche verschafften ihnen allerdings auch die Möglichkeit, den Belegschaften Veränderungen der Arbeitszeit- und Lohnsysteme aufzuzwingen, auch wenn deren Bedeutung angesichts der faktischen Widerstandsmöglichkeiten der Belegschaften während guter Konjunkturphasen jeweils beschränkt blieben.

Nach 1923 änderte sich die Situation grundlegend, da die Anpassungskrise die Unternehmen zu Leistungssteigerungen und Kostensenkungen zwang, und die Unternehmensleitungen gleichzeitig die Möglichkeit erhielten, das Arbeitsmarktrisiko zur Durchsetzung ihrer Zielvorstellungen zu nutzen. Die Belegschaften mußten sich - häufig in großen Auseinandersetzungen - diesen Vorgaben beugen, zumal auch die eigenen Zielvorstellungen mit dem Konjunkturumschwung wechselten. An die Stelle der Durchsetzung von Arbeitszeitverkürzungen und Lohnerhöhungen trat die Sicherung der Arbeitsplätze und die Aufrechterhaltung eines angemessenen Einkommensniveaus, wodurch Widerstände gegen Arbeitszeitverlängerungen und Leistungslohnsysteme zumindest teilweise entfielen, auch wenn letztere weiterhin als ungerecht empfunden wurden. Diese Konstellation dauerte bis zum Ende der Weltwirtschaftskrise an.

Für die betriebliche Mitbestimmung bedingte diese Entwicklung zwei ebenfalls grundverschiedene Phasen. Wurde betriebliche Mitbestimmung zwischen 1916 und 1923 von zahlreichen Belegschaften begrüßt und als Ansatzpunkt zu grundlegenden Veränderungen im Betrieb begriffen, so trat nach 1923/24 ihre defensive Funktion (Schutz der Rechte der Beschäftigten) in den Vordergrund. Beide Male erwies sich die durch das BRG kodifizierte Mitbestimmung als „Versager“. Während der offensiven Phase beschränkte sie die Handlungsmöglichkeiten der Belegschaftsvertreter, so daß die Belegschaften sich bei größeren wirtschaftlichen Kämpfen andere, nicht gesetzlich gebundene Vertretungen schufen, um nicht den Handlungsbeschränkungen des Gesetzes unterworfen zu sein, dessen Orientierung auf den Verhandlungskompromiß ein unbedingtes Durchsetzen der eigenen Forderungen ausschloß. In der defensiven Phase verhinderte wiederum die Beschränkung der betrieblichen Mitbestimmung auf soziale Fragen ihr Versagen ge- 
genüber den Folgen wirtschaftlicher Strukturveränderungen namentlich während der Rationalisierung. Die Belegschaften wurden auf diese Weise jeweils von den Möglichkeiten der betrieblichen Mitbestimmung enttäuscht und drückten diese Enttäuschung in der Wahl von Betriebsvertretern aus, die die betriebliche Mitbestimmung nur als Ansatzpunkt weitergehender Gesellschaftsveränderungen nutzen wollten.

Auf der anderen Seite wurde auch die Einstellung der Arbeitgeber zur betrieblichen Mitbestimmung konjunkturell bestimmt, aber nicht direkt, sondern über die Art der Nutzung der betrieblichen Mitbestimmung durch die Belegschaften. In der offensiven Phase der Belegschaften plädierten die Arbeitgeber daher für eine strenge Einhaltung des Betriebsrätegesetzes und verurteilten die Versuche der Belegschaften, das Gesetz zu instrumentalisieren. Nach 1923/24 ging das Interesse am Betriebsrätegesetz zurück und erwachte nur kurzfristig wieder, als sich seit 1929 eine erneute Politisierung der Betriebe abzuzeichnen schien. Eine Ausnahme bildete die Schwerindustrie, die das Gesetz durchweg ablehnte, zunächst wegen der Möglichkeit, das Gesetz politisch zu nutzen, später wegen seiner kostenträchtigen Wirkungen für die Ausgestaltung der Arbeitszeit- und Lohnsysteme, wobei die Strukturkrise, die in anderen Bereichen der deutschen Wirtschaft nicht existierte, eine zentrale Rolle spielte.

Die ökonomischen Rahmenbedingungen während der Weimarer Zeit bedingten daher eine strukturelle Überforderung des Betriebsrätegesetzes. Rahmenbedingungen, die einen dauerhaften und erfolgreichen Prozeß der Kooperation ermöglicht hätten, existierten zu keinem Zeitpunkt während der Weimarer Republik. Ökonomisch schwierige Rahmenbedingungen erzwingen aber nicht automatisch eine scharfe Konfrontation in den Betrieben, vor allem verlangen sie nicht zwangsläufig nach einem Versagen der Kommunikation.

2. Für die Art und Weise der betrieblichen Kommunikation in den untersuchten Branchen waren Betriebsführungstraditionen, Organisationsstrukturen und Belegschaftsverhalten von entscheidender Bedeutung. Dabei stellte sich heraus, daß zwischen den Farbenfabriken in Leverkusen und der späteren Abteilung Bergbau der VSt. deutliche Unterschiede existierten, und zwar insbesondere bei Betriebsführungstraditionen und Organisationsstrukturen. Das Belegschaftsverhalten wies mit einer hohen Quote ungelernter Arbeiter, einer hohen Fluktuation und einer Neigung zur „direkten Aktion“ bei gleichzeitiger geringer Gewerkschaftsorientierung hingegen Ähnlichkeiten auf.

Die unterschiedlichen Betriebsführungstraditionen und die verschiedenen Organisationsstrukturen waren vor allem eine Folge der Produktionstechnik, der damit verbundenen Struktur der Produktionsprozesse und der notwendigen Qualifikation des leitenden Personals. Im Bergbau handelte es sich mehr oder weniger um Einproduktbetriebe mit klarer, in der Linie organisierter Produktionsprozesse, die stabförmig nur im Bereich der Bergtechnik und der Betriebswirtschaft ausdifferenziert waren. Die Chemiefabrik in Leverkusen war hingegen eine hochkomplexe Vielproduktfabrik mit zahlreichen Produktionsstufen, einer hiervon getrennten Instandhaltungsabteilung und einer großen Forschungsabteilung. Während der Bergbau keiner flexiblen Organisationsstruktur bedurfte, um unterschiedliche Funktionsabteilungen zu integrieren, benötigten die Farbenfabriken 
ein differenziertes Ausschuß- und Kommunikationssystem, um die arbeitsteilig aufeinander verwiesenen Fabrikteile integrieren und koordinieren zu können. Klassischen Organisationskonzepten entsprechend könnte man die Abteilung Bergbau der VSt. als autoritäre, starre Unternehmensorganisation, die Farbenfabriken in Leverkusen als technokratisch-flexible Organisationsform bezeichnen.

Derartige Unternehmensorganisationen besitzen jeweils unterschiedliche Reaktionsweisen auf eine hohe Veränderungsdynamik in ihrer Umwelt. Während technokratisch-flexible Strukturen sich durch interne Differenzierung auf äußere Änderungen einstellen, tendieren starre Organisationsformen dazu, die Veränderungsdynamik in der Umwelt zu bekämpfen, um die eigenen Organisationsstrukturen aufrechterhalten zu können. Diese von der Organisationslehre aufgestellte These wird durch die betrieblichen Fallstudien bestätigt. Während in Leverkusen die Herausforderungen der zweiten Kriegshälfte und der Revolution durch Organisationsanpassungen erleichtert und über eine neue Kommunikationsstruktur die Vorschriften der neuen Betriebsverfassung in die bisherigen Organisationsstrukturen eingebaut wurden, blieben die wirtschaftlichen und politischen Herausforderungen im Ruhrbergbau ohne organisatorische Folgen für die Bergbauunternehmen. Die in der Unternehmensumwelt eingetretenen Änderungen fanden mithin im Bergbau keine Entsprechung in internen Differenzierungsprozessen. Statt dessen wurden die scheinbar erprobten Organisationsformen mit Macht aufrecht erhalten.

Die betriebliche Mitbestimmung traf in Leverkusen und im Ruhrbergbau daher auf unterschiedliche Voraussetzungen, die bereits zuvor in den technisch determinierten Organisationsstrukturen angelegt waren. Gleichwohl spielte das konkrete Verhalten der Unternehmensleitung seit der zweiten Kriegshälfte eine ebenso wichtige Rolle. In Leverkusen erfolgte eine gleitende, in den Worten Carl Duisbergs "opportunistische“ Anpassung an die neuen Rahmenbedingungen außerordentlich schnell. Im Bergbau reagierten die Zechenleitungen mit Verhärtungen der bisherigen Standpunkte und waren nicht bereit, diese den Veränderungen anzupassen. Abgesehen von den weltanschaulichen und sozialen Überzeugungen der Zechenleitungen und den organisationsstrukturell bedingten Defiziten in der Selbstbeobachtung beim Bergbau waren hierfür vor allem die konkreten Interaktionsprozesse seit der zweiten Kriegshälfte verantwortlich, die im Bergbau bereits im November 1918 Eskalationsstufen erreichten, die in Leverkusen erst zwei Jahre später eintraten. Der Grund hierfür war zweifellos der Zusammenbruch der öffentlichen Ordnung im Ruhrgebiet, während in Leverkusen die englische Besatzungsmacht die öffentliche Ordnung garantierte. Daß eskalierende Konfliktverläufe auch in Leverkusen zu Verhärtungen bei der Werksleitung führten, zeigte der Streikverlauf im Frühjahr 1921. Sie begannen allerdings erst, als der organisatorische Differenzierungsprozeß bereits abgeschlossen war, während im Ruhrbergbau Konflikteskalation und organisatorische Anpassungszwänge zeitlich zusammenfielen. Diese Unterschiede bedingten, bei allen Differenzen persönlicher und sozialer Art zwischen den jeweiligen Unternehmensleitungen, die unterschiedliche Bereitschaft der jeweiligen Manager, sich den neuen Verhältnissen anzupassen oder sie zu bekämpfen. 
Durch die Art und Weise der unternehmensinternen Verarbeitung der Herausforderungen der Jahre 1916 bis 1920 wurden daher wesentliche Weichen für die betriebliche Kommunikation gestellt. Während die Betriebsführungstraditionen des Bergbaus betriebliche Mitbestimmung ausschlossen, und die Organisationsstruktur der Unternehmen nicht hierarchische Kommunikation faktisch verunmöglichte, erwies sich die Betriebsführungstradition in Leverkusen als veränderungsoffen, die Organisationsstruktur als flexibel, freilich in den Grenzen, die durch die marktvermittelten wirtschaftlichen Rahmenbedingungen des Unternehmens gezogen wurden. Gleichwohl reichen die Faktoren wirtschaftliche Lage und Organisationsstruktur zur Erklärung der betrieblichen Konfliktverläufe nicht aus. Entscheidend war das Fehlen von Vertrauen in das Konfliktverhalten der Gegenseite, die Angst, durch Mitbestimmung mehr zu verlieren, als gewonnen werden konnte.

3. Der Zusammenbruch der betrieblichen industriellen Beziehungen nach 1916 war schließlich eng mit dem Verlust von Vertrauen in die Gegenseite und die Regeln des gegenseitigen Umgangs verbunden. Der Verhaltensopportunismus war dabei das entscheidende Moment der Vertrauenszerstörung, das durch eine Steigerung eigenmächtiger Kontrollansprüche ausgeglichen werden sollte. Derartige Kontrollansprüche formulierten die Arbeitgeber gegenüber der Arbeitsleistung und dem betrieblichen Verhalten ihrer Belegschaften, die Arbeiter gegenüber der Lohn- und Arbeitszeitpolitik der Werksleitungen oder der Verkaufs- und Preispolitik der Einzelhändler. Jede Seite unterstellte der anderen die Neigung zum Betrug, wobei der eigene Betrug jeweils moralisch gerechtfertigt erschien, da er aus sozialen oder wirtschaftlichen Zwängen herrühre. In den Beziehungen zwischen Kapital und Arbeit konnte der Mitte des Krieges beginnende Vertrauensverlust schließlich nicht mehr aufgehalten werden; Tarifsystem und betriebliche Mitbestimmung reichten nicht aus, um die Kompromißbereitschaft der jeweiligen Gegenseite in einer Weise zu dokumentieren, daß an ihr das eigene Verhalten konditioniert wurde. An die Stelle von Vertrauen trat Mißtrauen, an die Stelle von Verhaltenserwartungen Kontrollansprüche, die der jeweiligen Gegenseite schließlich jede eigenständige Handlungsmöglichkeit nehmen sollten. Das allgegenwärtig werdende Mißtrauen wurde zugleich politisch und moralisch aufgeladen, wobei insbesondere die linksradikalen Teile der Arbeiterbewegung das Mißtrauen nutzten, um jede Kompromißbereitschaft auf Seiten der Arbeitgeber („Schwindel“) wie der Arbeitnehmer („Klassenverrat“) zu denunzieren.

Im Rahmen der wirtschaftlichen und sozialen Krise bei gleichzeitig nur beschränkt funktionierenden unternehmensinternen Kommunikationsprozessen können der Vertrauensverlust, das sich verbreitende Mißtrauen und die Hoffnung auf die eigenmächtige Kontrollgewalt als Ursachen der Behinderung funktionierender Mitbestimmungsprozesse kaum überschätzt werden. Vor allem zwischen 1918 und 1923 verhinderte diese Konstellation hoher Kampfbereitschaft und geringer Kommunikationsdichte bei abgrundtiefem gegenseitigen Mißtrauen die Entstehung, Erprobung und Bewährung funktionierender betrieblicher Mitbestimmungsstrukturen. An dieser Konstellation scheiterte auch die staatliche Mitbestimmungspolitik der frühen Nachkriegsjahre. Weder konnte der Staat von sich aus die wirtschaftliche Krise bekämpfen, noch war er dazu in der Lage, das gegen- 
seitige Mißtrauen gleichsam per Verordnung zu beseitigen. Das sich im Nachhinein zeigende, entscheidende Defizit der Betriebsrätegesetzgebung, der Verzicht darauf, die Mitbestimmungsstrukturen in der Unternehmensorganisation zu verankern, war zudem nach 1920 nicht mehr zu beseitigen, nachdem bereits zuvor alle Ansätze politisch gescheitert waren, die auf eine Ausdifferenzierung der Unternehmensstrukturen über die Aufsichtsratsbeteiligung der Betriebsräte hätten hinauslaufen können. Nach 1920 aber wurde dieses Defizit lediglich von den nichtsozialistischen Angestelltenorganisationen gesehen und seine Beseitigung angemahnt. Solange die abhängig Beschäftigten kaum Einfluß auf das wirtschaftliche Wohlergehen ihrer Betriebe hatten, waren sie am Betriebswohl nicht dauerhaft zu interessieren, argumentierten GDA und Werkmeisterverband, womit sie de facto die Mitbestimmungsgesetzgebung des Montanmitbestimmungsgesetzes der frühen fünfziger Jahre zumindest programmatisch vorwegnahmen. Für alle anderen interessierten Gruppen aber schien nach 1923/24 kein Handlungsbedarf mehr zu bestehen, zumindest nicht in Richtung einer Erweiterung der betrieblichen Mitbestimmung hin zur Unternehmensmitbestimmung. Nicht zuletzt deshalb unterblieben im politischen Raum entsprechende Initiativen, und das Reichsarbeitsministerium konnte angesichts der Unbeweglichkeit der politischen Fronten zu Recht behaupten, erst die Zeit werde über den Erfolg oder Mißerfolg des Betriebsrätegesetzes entscheiden. Rationalisierung und Weltwirtschaftskrise untergruben schließlich den Stellenwert des Betriebsrätegesetzes in einer Weise, daß sein Wegfall nach 1933 kaum Aufsehen erregte, geschweige denn betriebliche Auswirkungen hatte.

Eine Sicherung der betrieblichen Mitbestimmung hätte dreierlei verlangt. Zunächst Zeit und in der Zeit einigermaßen kalkulierbare wirtschaftliche Rahmenbedingungen, zweitens differenzierte betriebliche Kommunikationsstrukturen und schließlich drittens Vertrauen der betrieblichen Akteure in das nüchterne Verhalten ihrer jeweiligen Konfliktpartner. Gesetzlich hätten die betrieblichen Kommunikationsstrukturen präziser geregelt werden können, doch hätte der Gesetzgeber weder berechenbare wirtschaftliche Rahmenbedingungen noch das Regelvertrauen der Konfliktpartner durchsetzen können. Bei Vorliegen dieser drei Faktoren hätte sich im Laufe der Zeit eine veränderte, sozialintegrative neue Governance-Struktur ${ }^{17}$ in den Unternehmen entwickeln können, für die es in den Leverkusener Farbwerken auch bereits die ersten Anzeichen gab. So wie die Entwicklung in Weimar aber praktisch verlief, gab es keine Möglichkeit, die ökonomischen und sozialen Vorteile der neuen betrieblichen Mitbestimmung zu verwirklichen. Sozialpartnerschaft hatte keine realistische Chance, zur Leitreferenz des Verhaltens der betrieblichen Akteure zu werden und - im Siegenthalerschen Sinne - neues Regelvertrauen, also routinisierte Strukturen konsensualer Weltkonstruk-

17 Da es sich bei der vorliegenden Arbeit nicht um eine theoretische Untersuchung handelt, können und sollen die vorliegenden Befunde auch nicht ausführlich theoretisch interpretiert werden. Sichtbar geworden sein dürfte freilich, daß eine Governance-Struktur kein intentional gewolltes System der Koordination von Arbeitsteilung darstellt, sondern selbst ein nur historisches begreifbares Produkt ist, dessen Entstehen sich nicht den Willensakten einzelner Akteure verdankt, auch wenn eine erfolgreiche Struktur natürlich viele Väter hat, die das, was eintrat, selbstverständlich auch gewollt zu haben vorgeben, als es noch nicht eingetreten war. Dies dürfte ein theoretisch zumindest beachtenswertes Ergebnis der historischen Betrachtung sein. 
tion zu stiften, die von allen Beteiligten unabhängig von ihren materiellen Konflikten im Grundsatz akzeptiert worden wären. Die Zerstörung von sozialem und arbeitsorganisatorischem Regelvertrauen zwischen 1914 und 1918 mündete in der Republik keineswegs in einen Prozeß der Rekonstituierung von Verfahrensweisen, sondern unter deren spezifischen Bedingungen in einen Kampf um die Festlegung von Verfügungsrechten, der keinen konsensualen Ausweg hatte. Die Revolution schuf, wenn überhaupt, so nur das Vertrauen dahingehend, daß Mißtrauen angemessen erschien. Allein diese Tatsache, daß nämlich die zeitgenössischen Akteure die Ausweglosigkeit ihrer materiellen Konflikte nicht begriffen und es ihnen nicht gelang, sich statt sinnlosen Streits auf gemeinsame Verfahrensweisen zu verständigen, die ein Regulieren der Konflikte erlaubt hätten, mag im Nachhinein den Beobachter befremden, doch teilen diese kombattantenhafte Haltung noch heute die meisten Historiker selbst, ganz abgesehen davon, daß auch in der gegenwärtigen Welt Verfahrensverständigungen und die Orientierung an Systemrationalität regelmäßig von simplen Partikularismen zerstört werden. Insofern scheiterte die betriebliche Mitbestimmung in der Weimarer Republik auch nur zum Teil an zeitbedingten Faktoren; im Grunde markiert ihr Scheitern die Unwahrscheinlichkeit nüchterner Verständigung schlechthin. 